\title{
Efficacy of Ulinastatin and Sulforaphane Alone or in Combination in Rat Model of Streptozotocin Diabetes Induced Vascular Dementia
}

\author{
Poonam Sharma ${ }^{1}$, Prachi Kaushik ${ }^{1}$, Swati Jain ${ }^{2}$, Brij Mohan Sharma ${ }^{2}$, Rajendra Awasthi ${ }^{3}$, Giriraj Thirupathirao \\ Kulkarni $^{3}$, Bhupesh Sharma ${ }^{1,4}$ \\ ${ }^{1}$ Department of Pharmacology, Amity Institute of Pharmacy, Amity University Uttar Pradesh, Noida, ${ }^{2}$ Department of Pharmacology, School of \\ Pharmacy, BIT, Meerut, ${ }^{3}$ Amity Institute of Pharmacy, Amity University Uttar Pradesh, Noida, ${ }^{4} \mathrm{CNS}$ and CVS Pharmacology, Conscience \\ Research, Delhi, India
}

\begin{abstract}
Objective: Vascular Dementia $(\mathrm{VaD})$, is associated with metabolic conditions. Diabetes is a major risk factor for the development of $\mathrm{VaD}$. This study investigates the efficacy of ulinastatin (UTI) and sulforaphane (SUL) in streptozotocin (STZ)-diabetes induced vascular endothelium dysfunction and related dementia.

Methods: Single dose STZ (50 mg/kg i.p.) was administered to Albino Wistar rats (male, $200-250$ g). Morris water maze and attentional set shifting tests were used to assess the spatial learning, memory, reversal learning, and executive functioning in animals. Body weight, serum glucose, serum nitrite/nitrate, vascular endothelial function, aortic superoxide anion, brains' oxidative markers (thiobarbituric acid reactive species-TBARS, reduced glutathione-GSH, superoxide dismutase-SOD, and catalase-CAT), inflammatory markers (IL-6, IL-10, TNF- $\alpha$, and myeloperoxidase-MPO), acetylcholinesterase activity-AChE, blood brain barrier (BBB) permeability and histopathological changes were also assessed. UTI $(10,000 \mathrm{U} / \mathrm{kg})$ and SUL $(25 \mathrm{mg} / \mathrm{kg})$ were used alone as well as in combination, as the treatment drugs. Donepezil (0.5 $\mathrm{mg} / \mathrm{kg}$ ) was used as a positive control.

Results: STZ-administered rats showed reduction in body weight, learning, memory, reversal learning, executive functioning, impairment in endothelial function, BBB permeability, increase in serum glucose, brains' oxidative stress, inflammation, AChE-activity, BBB permeability and histopathological changes. Administration of UTI and SUL alone as well as in combination, significantly and dose dependently attenuated the STZ-diabetes-induced impairments in the behavioral, endothelial, and biochemical parameters.

Conclusion: STZ administration caused diabetes and VaD which was attenuated by the administration of UTI and SUL. Therefore, these agents may be studied further for the assessment of their full potential in diabetes induced VaD.
\end{abstract}

KEY WORDS: Diabetes mellitus; Dementia; Cognition; Endothelium; Cholinesterase inhibitors.

\section{INTRODUCTION}

Vascular dementia $(\mathrm{VaD})$, the second most occurring form of dementia, is characterized by cognitive impairment, which is quantified as deficit in memory, cognition, executive function, language, attention and behavior [1]. $\mathrm{VaD}$ occurs as a result of impaired vascular system which disrupts the sufficient supply of blood and hence oxygen

Received: July 2, 2020 / Revised: September 25, 2020

Accepted: December 11, 2020

Address for correspondence: Bhupesh Sharma

Department of Pharmacology, Amity Institute of Pharmacy, Amity

University Uttar Pradesh, Sector-125, Noida, Uttar Pradesh

201313, India

E-mail: drbhupeshresearch@gmail.com

ORCID: https://orcid.org/0000-0002-3423-007X as well as nutrients to the brain that ultimately cause neuronal cell death [2]. It is observed that cerebral glucose utilization and energy metabolism are the abnormalities that precede the initial stages of cognitive impairment [3]. This has allowed the use of metabolic disorders associated animal models for understanding the efficacy of various therapeutics on behavioral as well as biochemical phenotypes observed in $\mathrm{VaD}$.

Diabetes is a metabolic disorder, includes impairment in the insulin action and signaling. Diabetes is well associated with increased cognitive dysfunction and risk of dementia in elderly life [4]. Studies report that, diabetes increases the probability of $\mathrm{VaD}$, both in early as well as late life stage of human [4]. Diabetes results into chronic

(ㄷ) This is an Open-Access article distributed under the terms of the Creative Commons Attribution Non-Commercial License (http://creativecommons.org/licenses/by-nc/4.0) which permits unrestricted non-commercial use, distribution, and reproduction in any medium, provided the original work is properly cited. 
hyperglycemia. Consistent hyperglycemia, cause endothelial dysfunction, blood-brain barrier (BBB) disruption, cerebral atrophy and declined cognitive abilities which are similar to those observed in Alzheimer disease (AD) and $\mathrm{VaD}$ [5]. Endothelial dysfunction hinders sufficient supply of blood to the brain. This limits the supply of required glucose and oxygen for proper functioning of the neuronal cells [3]. The brain studies of people suffering from dementia shows improper utilization of glucose as well as insensitivity to insulin causing damage to the neuronal cell. This is similar to diabetes [6]. Streptozotocin (STZ) administered rodents are being utilized as an animal model for diabetes [4]. STZ diabetes, increases oxidative stress and cause activation of proinflammatory biomarkers, which directly contribute to endothelial dysfunction [7]. In the last decade, we have extensively studied diabetes induced $\mathrm{VaD}$ in rats and successfully utilized diabetes induction as the experimental model of $\mathrm{VaD}$ [8-11].

Ulinastatin (UTI) a glycoprotein, is a serine protease inhibitor that, is isolated from a healthy human urine or is produced synthetically. UTI effect has been studied to improve the integrity of the BBB $[12,13]$. UTI has been reported to show protective effect in brain against traumatic brain injury [14], ischemia-reperfusion injury [15], brain edema [14], neurodegenerative disorder like Alzheimer's [16], Parkinson's and other psychiatric diseases [17]. UTI has improved learning, memory, spatial memory, cognitive dysfunction, and synaptic plasticity in rodent study $[18,19]$. Treatment with UTI suppresses early inflammation, microvascular and endothelial injury in brain [15,20]. UTI attenuates oxidative stress, inflammation and neural apoptosis in cerebral cortex of the rat $[16,21]$. Clinical and in vitro studies have also shown the protective effects of UTI against oxidative stress and inflammation [16,22]. Role of UTI has been studied for various brain conditions but the utility of UTI has not been studied in diabetes induced endothelial dysfunction and $\mathrm{VaD}$. We hypothesized that UTI, may provide beneficial effects in diabetes induced endothelial dysfunction and $\mathrm{VaD}$.

Sulforaphane (SUL) found in cruciferous vegetables, is a naturally occurring isothiocyanate that can cross the BBB easily after absorption [23]. SUL has been documented to show protective effects against several central nervous system (CNS) disorders such as cerebral ischemia, Parkinson's, Alzheimer's, schizophrenia, epilepsy, multiple sclerosis and autism [24,25]. SUL has been observed to reduce hyperglycaemia [26]. SUL has shown benefits against impairment in learning, memory, spatial memory, and cognitive function [27]. SUL improves the integrity if disrupted BBB [28] as well as inhibits microglial activation induced neuroinflammation [29]. The therapeutic or prophylactic effect of SUL in the CNS disorder is due to its protective effect against oxidative stress [30], inflammation [31] and neuronal death [32]. Role of SUL has been studied for various brain conditions but the utility of SUL has not been studied in diabetes induced endothelial dysfunction and $\mathrm{VaD}$. We hypothesized that SUL, may provide beneficial effects in diabetes induced endothelial dysfunction and $\mathrm{VaD}$.

In above view, we hypothesized that UTI and SUL, may provide beneficial effects in diabetes induced endothelial dysfunction and $\mathrm{VaD}$ and thus, the present study has been designed. In this study, we have induced diabetes in rodents and effects of UTI and SUL alone as well as in combination have been examined. Animals were assessed for learning, memory, vascular endothelial function, serum as well as brain biochemistry, BBB permeability and histopathological changes. Donepezil was utilised as a positive control [11].

\section{METHODS}

\section{Animals}

The present study employed adult male albino wistar rats weighing around 220-250 gm. Animals were housed and taken care of, until the completion of the study in the animal house of the institution, with free access to standard laboratory's pellet chow diet. Animals had an exposure to 12 hours. light and dark cycle allowing proper acclimatization to the laboratory conditions for a span of 5 days prior to the behavioral study. The experiments conducted were as per the study protocol approved by Institutional Animal Ethics committee (IAEC) between 9:00 and 16:00 hour maintaining semi sound proof environment in laboratory, following the guidelines provided by CPCSEA, Ministry of Environment and Forests, Government of India (no. 1147/ab/07/CPCSEA).

\section{Drugs and Chemicals}

UTI was obtained from Abbott laboratories., India. SUL has been obtained from Sun Pharma Pvt. Ltd., India. 5,5, 
dithiobis (2-nitrobenzoic acid) (DTNB), reduced glutathione (GSH), N-naphthyl ethylene diamine, bovine serum albumin (BSA), thiobarbituric acid were purchased from Sisco Research Laboratories Pvt Ltd., Mumbai, India. All the drug solutions were prepared freshly before use.

\section{STZ Administration}

Single dose of STZ (50 mg/kg i.p.) was administered to rats by dissolving in citrate buffer $(0.1 \mathrm{M} ; 10 \mathrm{ml} / \mathrm{kg}$, i.p.; $\mathrm{pH} 4.5)$ to induce experimental diabetes and related dementia [8-11].

\section{Experimental Protocol}

In the present study, total 14 groups have been employed with each group consisting of 8 male Wistar rats.

Group I - Control group: Animals were exposed to behavioral assessment from Day 1.

Group II - Saline: Rats were administered saline $(0.9 \%$ $\mathrm{w} / \mathrm{v} ; 10 \mathrm{ml} / \mathrm{kg}$, i.p.; daily) for 26 days with exposure to behavioral assessment from 22nd day onwards.

Group III - PBS: Rats were administered PBS $(10 \mathrm{ml} / \mathrm{kg}$, p.o.; $\mathrm{pH} 7.4$, daily) for 26 days with exposure to behavioral assessment from 22nd day onwards.

Group IV - Citrate buffer: Rats were administered a single dose of citrate buffer (0.1 M; $10 \mathrm{ml} / \mathrm{kg}$, i.p.; pH 4.5) after which they were exposed to behavioral assessment from 52nd day of its administration.

Group V - UTI (10,000 U/kg, i.p.), per se: Rats were administered UTI (10,000 U/kg, i.p., daily) for 26 days with exposure to behavioral assessment from 22nd day onwards [12].

Group VI - SUL (25 mg/kg, p.o.) per se: Rats were administered SUL (25 mg/kg, p.o., daily), for 26 days with exposure to behavioral assessment from 22nd day onwards [33].

Group VII - Donepezil (0.5 mg/kg, i.p.) per se: Rats were administered donepezil ( $0.5 \mathrm{mg} / \mathrm{kg}$, i.p., daily) for 26 days with exposure to behavioral assessment from 22nd day onwards. Donepezil serves as a positive control.

Group VIII - STZ: Rats were administered a single dose of STZ (50 mg/kg, i.p.) after which they were exposed to behavioral assessment from 52 nd day of its administration.

Group IX - STZ + UTI (10,000 U/kg, i.p.): UTI (10,000 $\mathrm{U} / \mathrm{kg}$, i.p.) was administered to the STZ (50 mg/kg, i.p.) diabetic rats, starting from day 30th of the STZ treatment to day 56th, with exposure to behavioral assessment from 52nd day onwards.

Group X - STZ + SUL (25 mg/kg, p.o.): SUL (25 mg/kg, p.o.) was administered to the STZ $(50 \mathrm{mg} / \mathrm{kg}$, i.p.) diabetic rats, starting from day 30th of the STZ treatment to day 56th, with exposure to behavioral assessment from 52nd day onwards.

Group XI - STZ + UTI (10,000 U/kg, i.p.) + SUL (25 $\mathrm{mg} / \mathrm{kg}$, p.o.) - UTI (10,000 U/kg, i.p.) + SUL (25 mg/kg, p.o.) in combination were administered to the STZ (50 $\mathrm{mg} / \mathrm{kg}$, i.p.) diabetic rats, starting from day 30th of the STZ treatment to day 56th, with exposure to behavioral assessment from 52nd day onwards.

Group XII - STZ + Donepezil (0.5 mg/kg): Donepezil (0.5 mg/kg, i.p.; daily) was administered to the STZ (50 $\mathrm{mg} / \mathrm{kg}$, i.p.) diabetic rats, starting from day 30th of the STZ treatment to day 56th, with exposure to behavioral assessment from 52nd day onwards.

During our previous investigations, we observed that hyperglycemia induced endothelial dysfunction was observed during 14-15th day (after 2 week). The behavioral deficits due to endothelial dysfunction became comparative after 3-4 weeks [7-11]. We therefore, allowed the induction of endothelial dysfunction and behavioral deficits with STZ administration and then started the treatments. All the treatments were started simultaneously. Behavioral assessments were initiated with the exposure of animals to the MWM for first five days followed by attentional set shifting test (ASST) for next 10 days. We have not aimed to perform a time-course experiment therefore, after the last day assessment of ASST, animals were sacrificed to remove thoracic aorta and brain for the biochemical parameters' estimation.

\section{Assessment of Spatial learning and Memory by Morris Water Maze}

Morris water maze [34] apparatus is used to test memory. It consists of large circular pool equally divided into four quadrants. The water was filled and was made opaque using colored dye. White painted platform was placed in the target quadrant, $1 \mathrm{~cm}$ below the water surface and the platform was left undisturbed aa through the training session.

Acquisition trial: The rat was allowed to explore and locate the submerged platform for 120 seconds and then allowed to stay on the platform for next 20 seconds. If it fails to find the platform within 120 seconds, it was then guid- 
ed gently to the platform. Each animal was subjected to four trials in the same session, for four consecutive days, with every trial having different drop position. After each trial animal was allowed to rest for 5 minutes. and the time utilized by the animal in locating the submerged platform in MWM was be noted down as Escape Latency Time (ELT) and it was regarded as the index for acquisition or learning.

Retrieval trial: after the acquisition trials, on the fifth day the platform was removed and the rat was placed in the pool to explore for 120 seconds. The mean time spent in the pool's four quadrants are observed, recorded and the mean time spent searching for the platform by the animal in target quadrant was jotted down as an index for retrieval. Experiments were conducted between 9:00 and 16:00 hour.

\section{Assessment of Cognitive Function Using Attentional Set Shifting Test}

ASST model is used for measuring cognitive function. ASST works on the tendency of the animals to dig vigorously in to their bedding. The digging procedure consist of the habituation, training and testing paradigm. A week before training and testing period, animals were food restricted so that $85 \%$ free-fed body weight could be maintained. This was followed by habituation and training from day 1 to day 7 . On day 8 and day 9 , animals were allowed to learn discrimination between the dimensions of digging medium and the respective odor. On day 10 (testing paradigm), animals were tested on a series of discrimination stages, simple discrimination (SD), compound discrimination (CD), reversal 1 (REV 1), intra-dimensional (ID), reversal 2 (REV 2), extra dimensional (ED) and reversal 3 (REV 3) stages presented in a fixed order for all animals [35]. Biasness was prevented by carrying forward all the behavioral experiments manually by the colleague, who was unknown to the experimental protocol.

\section{Assessment of Vascular Endothelial Function Using Rat Aortic Ring Preparation}

For the assessment of vascular endothelial function, the rats underwent cervical dislocation after which the rats were decapitated to remove thoracic aorta. After removal, the thoracic aorta was cut into rings of around 4 to $5 \mathrm{~mm}$ width [34]. Aortic rings prepared were suspended and mounted to the organ chamber with the help of two stain- less clips. Organ bath contained Krebs's buffer with composition (in $\mathrm{mM}$ ): $\mathrm{NaCl}, 118.3 ; \mathrm{KH}_{2} \mathrm{PO}_{4}, 1.2 ; \mathrm{KCl}, 4.7$; $\mathrm{CaCl}_{2}$, 2.5; $\mathrm{MgSO}_{4}, 0.6 ; \mathrm{NaHCO}_{3}, 25.0 ;$ EDTA, 0.026; pH 7.4 at $37^{\circ} \mathrm{C}$ and gassed with $95 \% \mathrm{O}_{2}$ plus $5 \% \mathrm{CO}_{2}$. A tension of $1.5 \mathrm{~g}$ was given to the aorta ring for the time span of 90 minutes in the Krebs's solution which, was changed after every 15 minutes. Upon equilibrium, aortic rings were challenged again with $\mathrm{KCl}(60 \mathrm{mM})$ followed by washing and equilibration period of 30-minute. After this, the contractile response was elicited by the addition of phenylephrine $(1 \mu \mathrm{M})$ and when the plateau of contraction was reached, Ach or SNP was added to the organ bath for inducing endothelium-dependent or-independent relaxation.

\section{Biochemical Parameters}

\section{Collection of samples}

For biochemical estimation, blood samples were collected just prior to the animal sacrifice and were kept for 30 minutes at room temperature followed by centrifugation for 15 minutes at 4,000 rpm to separate the serum which was finally used to estimate serum glucose and nitrite/nitrate concentration.

For biochemical estimations in the brain tissue, the animals were firstly sacrificed to remove the brains. The animals were decapitated and with the help of the scalpel or razor blade, a single cut along the lengthwise was made all the way starting from the point between the eyes to the posterior aspect. With the help of ronguers, pull back the skin and the muscles to expose the skull underneath. Thick neck musculature present at the base of the skull was removed to see the entire posterior aspect of the brain. Now, when the skull is exposed, the bits of the skull was peeled away from the base surface using ronguers. When the posterior portions of the skull were removed the other dorsal portions come off in three large portions. The exposed brain is extracted and then the brains were homogenized using Teflon homogenizer using phosphate buffer $(10 \% \mathrm{w} / \mathrm{v}, \mathrm{pH} 7.4)$ with protease inhibitor $(0.1 \mathrm{ml}$, $1 \mathrm{X})$ in it, followed by centrifugation for 15 minutes at 3,000 rpm to obtain clear supernatant [36].

\section{Estimation of serum glucose}

To ascertain the induction of diabetes, fasting serum glucose levels were monitored every week spectrophotometrically (UV-1800 ENG 240 V, Shimadzu Corporation, 
Kyoto, Japan) at $550 \mathrm{~nm}$, using commercially available kit (Reckon Diagnostics Pvt. Ltd., New Delhi, India) by glucose oxidase peroxidase (GOD-POD) method [10]. Blood was collected from the orbital sinus post anesthesia by Pasteur pipette. Rats with fasting serum glucose levels greater than $200 \mathrm{mg} / \mathrm{dl}$ were considered diabetic. The animals that did not become diabetic were excluded from the study $[37,38]$.

\section{Estimation of serum nitrite/nitrate concentration}

The measurement of serum nitrite/nitrate concentration helps in the assessment of nitric oxide levels in the peripheral blood supply. The nitric oxide levels serve as an index for the endothelial function in the blood vessels. For the assessment of serum nitrite concentration, $400 \mu \mathrm{l}$ of carbonate buffer ( $\mathrm{pH}$ 9.0) was added to $100 \mu \mathrm{l}$ of standard solution or serum with further addition of $0.15 \mathrm{~g}$ of copper-cadmium alloy. The tubes were allowed to incubate at room temperature for a period of 1 hour to cause the reduction of nitrate into nitrite. This reaction was stopped with the addition of $100 \mu \mathrm{l}$ of $0.35 \mathrm{M}$ Sodium hydroxide. This was followed by the addition of $400 \mu \mathrm{l}$ of zinc sulphate solution $(120 \mathrm{mM})$ to cause deproteination of samples. The samples were left to stand still for 10 minutes and then was centrifuged at 4,000 g for next 10 minutes. Finally, to the aliquots $(500 \mu \mathrm{l})$ of clear supernatant, Griess reagent (250 $\mu$ l of $1.0 \%$ sulphanialamidepreparedin $3 \mathrm{~N} \mathrm{HCl}$ and $250 \mu \mathrm{l}$ of $0.1 \% \mathrm{~N}$-naphthylethylenediamine prepared with water) was added and serum nitrite/nitrate concentration was measured at $545 \mathrm{~nm}$ spectrophotometrically (UV-1800 ENG 240V, Shimadzu Corporation) [34]. Sodium nitrite $(5-50 \mu \mathrm{M})$ standard curve to calculate the serum nitrite/nitrate concentration, was plotted.

\section{Estimation of aortic production of super oxide anion}

The superoxide anion in aorta suggest the oxidative stress in the aorta. For the assessment of superoxide anion levels, $10 \mathrm{~mm}$ transverse rings of sliced aorta were placed for 1.5 hours. in nitrobluetetrazolium $(100 \mathrm{mM} / \mathrm{L})$ containing buffer $(5 \mathrm{ml})$ at $37^{\circ} \mathrm{C}$. The reduction of the nitro blue tetrazolium (NBT) was brought to halt by the addition of $5 \mathrm{ml}$ of $\mathrm{HCl}(0.5 \mathrm{~mol} / \mathrm{L})$. The rings were then minced and homogenized in a mixture of $\mathrm{NaOH}(0.1$ $\mathrm{M} / \mathrm{L}), \operatorname{SDS}(0.1 \%)$, and $(40 \mathrm{mg}) / \mathrm{L}$ diethylentriaminepentaacetic acid in water. The resulted mixture underwent centrifugation for 2 minutes at 20,000 g to resuspend the re- sultant pellet in pyridine $(1.5 \mathrm{ml})$. The mixture was kept at $80^{\circ} \mathrm{C}$ to extract formazon for the span of 1.5 hours, post which, the mixture underwent centrifugation again for 10 minutes at $10,000 \mathrm{~g}$. The formazon absorbance was estimated at $540 \mathrm{~nm}$ spectrophotometrically [34]. The reduced NBT amount was calculated with the help of formula:

\section{Amount of reduced NBT $=\mathrm{A} \times \mathrm{V} /(\mathrm{T} \times \mathrm{M} \times \mathrm{e} \times \mathrm{I})$}

where $\mathrm{A}=$ absorbance,

$\checkmark$ is the volume of pyridine,

$\mathrm{T}=$ time when the rings were incubated in NBT,

$M=$ wet mass (blotted) of the aortic rings,

$\mathrm{e}=$ extinction coefficient $\left(0.71 \mathrm{~mm}^{-1}\right)$,

$\mathrm{I}=$ length of the light path.

\section{Estimation of brain total protein}

For the assessment of brain total protein, bovine serum albumin $0.2-2.4 \mathrm{mg} / \mathrm{ml}$ was used as a standard for plotting the standard curve [39]. Brains' total protein content was measured at $750 \mathrm{~nm}$ spectrophotometrically using standard curve of BSA. These values were expressed as $\mathrm{mg} / \mathrm{ml}$ of supernatant.

\section{Assessment of brain lipid peroxidation}

The whole brain thiobarbituric acid reactive substances (TBARS) levels serves as a marker of lipid peroxidation in the brain. For the assessment of brain TBARS levels, 0.2 $\mathrm{ml}$ of supernatant of the homogenate was pipette out in a test tube into which, $0.2 \mathrm{ml}$ of $8.1 \%$ sodium dodecylsulphate, $1.5 \mathrm{ml}$ of $30 \%$ acetic acid (pH3.5), $1.5 \mathrm{ml}$ of $0.8 \%$ of thiobarbituric acid and distilled water were added, to make the volume up to $4 \mathrm{ml}$. The test tubes were incubated for 1 hour at $95^{\circ} \mathrm{C}$, and then were cooled allowing the addition of $1 \mathrm{ml}$ of distilled water, and $5 \mathrm{ml}$ of n-butanol-pyridine mixture $(15: 1 \mathrm{v} / \mathrm{v})$. The tubes were centrifuged at 4,000 g for 10 minute. The absorbance of developing pink color was measured spectro- photometrically at $532 \mathrm{~nm}$ [39]. A standard calibration curve with the use of 1-10 nM of 1,1,3,3-tetramethoxypropane was prepared. The TBARS values were expressed as nano moles/mg of protein.

\section{Assessment of brain glutathione (GSH) levels}

Whole brain GSH levels help in the assessment of 
brains' endogenous antioxidant GSH levels. For the assessment of GSH levels, after the halogenation process, the supernatant formed was centrifuged at 1,000 $\mathrm{g}$ for 40 minutes at $4^{\circ} \mathrm{C}$ after mixing in 1:1 ratio with trichloroacetic acid $(10 \% \mathrm{w} / \mathrm{v})$ in tubes. The supernatant formed after the centrifugation was further mixed with $2 \mathrm{ml}$ of disodium hydrogen phosphate $(0.3 \mathrm{M})$ with further addition of $0.25 \mathrm{ml}$ of $0.001 \mathrm{M}$ freshly prepared 5,50-dithiobis (2-nitrobenzoicacid) (DTNB dissolved in 1\%w/v sodium citrate). The absorbance was estimated at $412 \mathrm{~nm}$ spectrophotometrically (UV-1800 ENG 240V, Shimadzu Corporation). A standard curve with the use of $10-100$ $\mu \mathrm{M}$ of glutathione (reduced form) was plotted and the values were expressed as micromoles of reduced glutathione/mg of protein [34].

\section{Assessment of brain superoxide dismutase (SOD) activity}

Whole brain SOD activity estimation helps in the assessment of brains' endogenous antioxidant SOD levels. Brain SOD activity was measured using spectrophotometrically (UV-1800 ENG 240V, Shimadzu Corporation) at $560 \mathrm{~nm}$ [35]. The method is based on the formation of water insoluble blue from the NBT reduction. For the estimation of SOD activity, brain homogenate $(0.5 \mathrm{ml})$ was mixed with $50 \mathrm{mM} \mathrm{Na}{ }_{2} \mathrm{CO}_{3}(1 \mathrm{ml}), 24 \mu \mathrm{m} \mathrm{NBT}(0.4 \mathrm{ml})$, and $0.1 \mathrm{mM}$ EDTA $(0.2 \mathrm{ml})$. To this mixture, $1 \mathrm{mM}$ hydroxylamine hydrochloride $(0.4 \mathrm{ml})$ was further added to begin the reaction. The blue colour thus developed in the reaction was observed at $560 \mathrm{~nm}$. Absorbance, at $560 \mathrm{~nm}$ and at $25^{\circ} \mathrm{C}$ was recorded at Zero-time and then every 30 seconds during the span of 5 minutes. Control reading was obtained by performing the above reaction excluding the brain homogenate. The rate of absorbance units $(A)$ increase per minute for the test and control sample(s) were recorded. The percentage inhibition of the NBT reduction served as a measure for the SOD presence and this was calculated with the help of the following formula:

$\%$ inhibition $=\{(\triangle \mathrm{A} 560 \mathrm{nM} / \mathrm{min})$ control $(\Delta \mathrm{A} 560 \mathrm{nM} / \mathrm{min})$ test $/(\Delta \mathrm{A} 560 \mathrm{nM} / \mathrm{min})$ control $\} \times 100$

where $(\triangle \mathrm{A} 560 \mathrm{nM}$ at 5 minutes and 30 seconds $-\mathrm{A} 560$ $\mathrm{nM}$ at 30 seconds $) / 5$ minutes $=\Delta \mathrm{A} 560 \mathrm{nM} /$ minute.

SOD activity in units represented the quantity of enzyme required to inhibit $50 \%$ of the NBT reduction and was expressed as units per mg of protein.

\section{Assessment of brain catalase (CAT) activity}

Whole brain CAT activity estimation helps in the assessment of brains' endogenous antioxidant CAT levels. For the assessment of catalase activity in the brain, $1 \mathrm{ml}$ brain homogenate was mixed with $1.9 \mathrm{ml}$ of phosphate buffer $(50 \mathrm{mM})$ maintained at $\mathrm{pH}$ 7.4. Further, addition of $1 \mathrm{ml} \mathrm{H} \mathrm{O}_{2}$ (30 mM) to the mixture initiated the reaction. The above mixture devoid of the brain homogenate serves as a blank. The decrease in the absorbance as a result of $\mathrm{H}_{2} \mathrm{O}_{2}$ decomposition was measured spectrophotometrically (UV-1800 ENG 240V, Shimadzu Corporation) at $240 \mathrm{~nm}$ against the blank [35]. Units were expressed in terms of quantity of enzyme required to decompose $1 \mu \mathrm{M}$ of $\mathrm{H}_{2} \mathrm{O}_{2} /$ minutes at $25^{\circ} \mathrm{C}$ and the activity was demonstrated as units/mg of proteins.

\section{Assessment of brain inflammatory markers}

Interleukin- IL-6, IL-10 and tumour necrosis factor alpha (TNF- $\alpha$ ) were assessed as neuro inflammatory markers in brain using RayBio ${ }^{\circledR}$ Rat ELISA kits. All three kits work on the principle of sandwich enzyme-linked immunosorbent assay (in vitro) for the quantifying rats' IL-6, IL-10, and TNF- $\alpha$ in supernatants at $450 \mathrm{~nm}$ on a 96 -well plate reader. The values were expressed in $\mathrm{pg} / \mathrm{ml}$ [40].

\section{Assessment of myeloperoxidase activity (MPO)}

The assessment of whole brain MPO levels serves as an inflammatory marker in the brain. For the estimation of brain MPO brain tissues were homogenized in 10 volumes $50 \mathrm{mmol} / \mathrm{L}$ phosphate buffer having $0.5 \%$ hexadecyl trimethyl ammonium bromide (HTAB) ( $\mathrm{pH} 6.0$ ). The homogenized samples underwent three simultaneous cycles of freezing and thawing later to which they were centrifuged for about 10 minutes at $10,000 \mathrm{~g}$ at $4^{\circ} \mathrm{C}$. The supernatant produced, was added with $1.9 \mathrm{ml}$ of $10 \mathrm{mM}$ phosphate buffer (pH6.0) and $145 \mu \mathrm{l}$ of $1.5 \mathrm{~mol} / \mathrm{L}$ o-dianisidine hydrochloride, containing $0.0005 \% \mathrm{w} / \mathrm{v}$ hydrogen peroxide and the change in the absorbance after the addition was recorded spectrophotometrically (UV-1800 ENG 240V, Shimadzu Corporation) at $450 \mathrm{~nm}$ for each sample [39]. Sample MPO activity was calculated from the reaction curve slope based on the equation: 
Specific activity $\left(\mu \mathrm{mol} \mathrm{H}_{2} \mathrm{O}_{2} / \mathrm{min} / \mathrm{mg}\right.$ protein $)=$ $\Delta$ OD per min/ $\Delta$ OD per $\mu$ mol $\mathrm{H}_{2} \mathrm{O}_{2} \times$ mg protein.

\section{Estimation of brain acetyl cholinesterase (AChE) activity}

The estimation whole brain AChE activity method involves the formation of thiocholine, during the rection between brain cholinesterase and the acetylthiocholine iodide. The thiocholine formed undergoes reaction with DTNB to produce yellow colour. $0.5 \mathrm{ml}$ supernatant liquid from the brain homogenate is pipetted out into volumetric flask of $25 \mathrm{ml}$ and is diluted with DTNB (5,5'-dithiobis-[2-nitrobenzoic acid]) (freshly prepared) solution $(10 \mathrm{mg})$ in Sorenson phosphate buffer $(100 \mathrm{ml}, \mathrm{pH} 8.0)$. After this, 2 portions of the mixture $(4 \mathrm{ml}$ each) is separated and poured into two different test tubes, from which one test tube is further added with 2 drops of eserine solution. However, to both test tubes, substrate solution (1 $\mathrm{ml}$ ) (acetylcholine iodide [75 mg] per distilled water [50 $\mathrm{ml}]$ ) is added. The test tube added with eserine is taken as blank while the absorbance change per min. of the sample (test) is measured at $420 \mathrm{~nm}$ spectrophotometrically (UV-1800 ENG 240V, Shimadzu Corporation) [36]. Activity of the ACHE is estimated using the formula:

$$
\begin{aligned}
R= & (\delta \text { O.D. } \times \text { volume of Assay }) \\
& (E \times m g \text { of protein })
\end{aligned}
$$

Where, $R=$ rate at which ' $n$ ' mole of acetylcholine iodide hydrolyzed/minute/mg protein

$$
\delta \text { O.D. }=\text { change in absorbance } / \text { minute }
$$$$
\mathrm{E}=\text { Extinction coefficient }=(13600 / \mathrm{M} / \mathrm{cm})
$$

\section{Assessment of BBB permeability (Evans blue)}

The permeability of BBB is impaired in VaD. For the assessment of BBB permeability, $4 \%$ Evans blue $(4 \mathrm{ml} / \mathrm{kg}$ i.p.) was administered and was allowed circulate for the span of 120 minutes before measurement. The brains were removed and cerebral cortex were weighed. Post weighing, the brain was homogenized in PBS $(3.5 \mathrm{ml}, 0.1$ $\mathrm{mol} / \mathrm{L}, \mathrm{pH} 7.4)$ followed by centrifugation at $15,000 \mathrm{~g}$ for 30 minutes. The supernatant produced was treated with an equal volume of TCA. This was followed by an overnight incubation at $48^{\circ} \mathrm{C}$. The quantification of the extravasated Evans blue was done spectrophotometrically
(UV-1800 ENG 240V, Shimadzu Corporation) at $615 \mathrm{~nm}$ [34].

\section{Tissue Preparation for Histopathology}

For histology and subsequent analysis, the removed brain samples, were washed with $0.9 \%$ saline and then fixed in $4 \%$ paraformaldehyde. After the process of fixation, the brain samples were further processed by embedding in the paraffin wax. The brain samples then underwent coronal sectioning of thickness $50 \mu$ using a manual rotary microtome (INCO laboratories, Ambala, India), 6 slices per brain were selected for qualitative and quantitative visualization. To assess the cell viability and state, Nissl staining was used. The sections were further stained using $0.1 \%$ cresyl violet and luxol fast blue was used as a secondary stain. The sections were studied under an inverted microscope (CIB-100; Coslab, Haryana, India) and photographs were taken at $400 \times$ magnification [41]. In each of the chosen microscopic fields the neuronal count was manually done by an individual blinded to the whole study using Image J (National Institutes of Health, Bethesda, $M D, U S A)$ and the average of 6 sections for each animal was taken up for statistical analysis.

\section{Statistical Analysis}

The data is represented as mean \pm standard deviation. The data was analyzed using one-way ANOVA (sigma stat 12.5) followed by Bonferroni's post hoc test. The data for endothelial function was analyzed using repeated measures of ANOVA followed by Newman Keul's test. The results were considered statistically significant when $p<0.05$.

\section{RESULTS}

No significant differences were observed in drug per se and vehicle control groups on any of the parameters assessed.

\section{Effect on Body Weight and Serum Glucose Levels}

Compared to control animals, STZ diabetes produced a significant decrease in body weight $(p<0.05)$ and increase in serum glucose levels $(p<0.05)$. Treatment with UTI $(10,000 \mathrm{U} / \mathrm{kg}$, i.p.), SUL ( $25 \mathrm{mg} / \mathrm{kg}$ ) alone and in combination and Donepezil $(0.5 \mathrm{mg} / \mathrm{kg}$, i.p., daily) had no significant effect on STZ diabetes induced decrease in body weight as well as increase in serum glucose levels (Table 1). 
Table 1. Effect on body weight and serum glucose level

\begin{tabular}{|c|c|c|c|c|}
\hline \multirow{2}{*}{ Groups } & \multicolumn{2}{|c|}{ Body weight (in grams) } & \multicolumn{2}{|c|}{ Serum glucose (mg/dl) } \\
\hline & Basal value & Final value & Basal value & Final value \\
\hline CONTROL & $227 \pm 5.8$ & $316 \pm 5.1$ & $97.3 \pm 4.2$ & $99.6 \pm 3.71$ \\
\hline SAL & $223 \pm 5.1$ & $319 \pm 6.1$ & $96.4 \pm 3.5$ & $98.4 \pm 4.32$ \\
\hline PBS & $231 \pm 5.6$ & $314 \pm 5.7$ & $98.1 \pm 4.01$ & $99.9 \pm 4.78$ \\
\hline CB & $229 \pm 5.0$ & $315 \pm 5.9$ & $97.3 \pm 4.1$ & $98.4 \pm 3.97$ \\
\hline UTI & $225 \pm 5.3$ & $312 \pm 6.2$ & $98.2 \pm 3.77$ & $99.7 \pm 4.1$ \\
\hline SUL & $227 \pm 5.2$ & $309 \pm 5.8$ & $97.7 \pm 3.82$ & $98.7 \pm 6.64$ \\
\hline DON & $231 \pm 4.9$ & $307 \pm 5.8$ & $97.98 \pm 3.97$ & $97.5 \pm 4.21$ \\
\hline STZ & $234 \pm 5.8$ & $214 \pm 5.1^{\mathrm{a}}$ & $98.2 \pm 3.51$ & $307.3 \pm 5.11^{\mathrm{a}}$ \\
\hline $\mathrm{STZ}+\mathrm{UTI}$ & $222 \pm 5.1$ & $239 \pm 5.21^{\mathrm{a}}$ & $98.2 \pm 3.23$ & $273.8 \pm 4.48^{\mathrm{a}}$ \\
\hline STZ + SUL & $232 \pm 5.3$ & $241 \pm 5.06^{\mathrm{a}}$ & $97.3 \pm 3.89$ & $270.3 \pm 4.87^{\mathrm{a}}$ \\
\hline STZ + UTI + SUL & $234 \pm 4.9$ & $246 \pm 5.32^{\mathrm{a}}$ & $98.1 \pm 3.98$ & $266.9 \pm 4.64^{\mathrm{a}}$ \\
\hline $\mathrm{STZ}+\mathrm{DON}$ & $226 \pm 5.4$ & $223 \pm 5.9^{\mathrm{a}}$ & $97.9 \pm 3.32$ & $288 \pm 4.76^{\mathrm{a}}$ \\
\hline
\end{tabular}

Values are presented as mean \pm standard deviation. $\mathrm{n}=8$.

SAL, saline; PBS, phosphate-buffered saline; CB, citrate buffer; UTI, ulinastatin; SUL, sulforaphane; DON, donepezil; STZ, streptozotocin.

Basal values represent values as assessed on day 1 of the study, whereas final values represent values as assessed on day 1 of Morris Water Maze. Statistically analyzed using one-way ANOVA (sigma stat 12.5) followed by Bonferroni's post hoc test.

${ }^{\mathrm{a}} p<0.05$ vs. control group; Body weight: $\mathrm{F}(11,84)=574.777$, Serum glucose: $\mathrm{F}(11,84)=3228.958$.

\section{Effect on Learning and Memory Using MWM}

In MWM, control animals showed a significant decrease in day 4 ELT as compared to day 1 ELT, along with significant increase in day 5 TSTQ as compared to other quadrants, depicting effective learning and memory. STZ diabetic rats showed a significantly higher day 4 ELT whereas, a significantly lower day 5 TSTQ when compared to the control rats depicting reduced learning and memory. Treatment with UTI (10,000 U/kg, i.p.) and SUL $(25 \mathrm{mg} / \mathrm{kg}$ ) alone as well as in combination and donepezil $(0.5 \mathrm{mg} / \mathrm{kg}$, i.p., daily) decreased the day $4 \mathrm{ELT}$, ( $\mathrm{F}[11,84]=$ $113.474, p<0.05)$ as well as increased the day 5 TSTQ, $(F[11,84]=47.124, p<0.05)$ when compared to STZ diabetic rats, signifying the attenuation of STZ diabetes induced reduction in learning and memory (Fig. 1A, B).

\section{Effect on Reversal Learning and Executive Functioning Using ASST}

STZ diabetic rats, in comparison to the control group showed increased number of trials during REV1 and ED stage of ASST signifying impairment in the reversal learning and executive functioning. Treatment with UTI $(10,000$ $\mathrm{U} / \mathrm{kg}$, i.p.) and SUL $(25 \mathrm{mg} / \mathrm{kg})$ alone as well as in combination and donepezil $(0.5 \mathrm{mg} / \mathrm{kg}$, i.p., daily) attenuated STZ diabetes induced increase the number of trials during REV1 $(\mathrm{F}[11,84]=385.836, p<0.05)$ and ED stage $(\mathrm{F}$ $[11,84]=442.116, p<0.05)$ indicating attenuation of
STZ diabetes induced impairment in reversal learning and executive functioning (Fig. 2).

\section{Effect on Endothelium Dependent and Independent Relaxation}

Acetylcholine (ACh) and sodium nitroprusside (SNP) dose dependently produces endothelium dependent and independent relaxation in phenylephrine $\left(3 \times 10^{-6} \mathrm{M}\right)$ precontracted isolated rat aortic ring preparation, respectively in control rats. STZ diabetes significantly attenuated ACh induced endothelium dependent relaxation, however, no affect was observed on the SNP induced endothelium independent relaxation. Treatment with UTI $(10,000 \mathrm{U} / \mathrm{kg}$, i.p.) and SUL $(25 \mathrm{mg} / \mathrm{kg})$ alone as well as in combination and donepezil $(0.5 \mathrm{mg} / \mathrm{kg}$, i.p., daily) attenuated the effect of STZ diabetes on endothelial dependent relaxation, $(p<0.05)$. These interventions however, had no effect on the endothelium independent relaxation (Fig. 3A, B).

\section{Effect on Serum Nitrite/Nitrate, Aortic Superoxide Anion}

Compared to the control animals, STZ diabetic rats showed a decrease in serum nitrite/ nitrate and increase in aortic superoxide anion levels. Treatment with UTI (10,000 $\mathrm{U} / \mathrm{kg}$, i.p.) and SUL $(25 \mathrm{mg} / \mathrm{kg})$ alone as well as in combination and donepezil $(0.5 \mathrm{mg} / \mathrm{kg}$, i.p., daily) prevented 

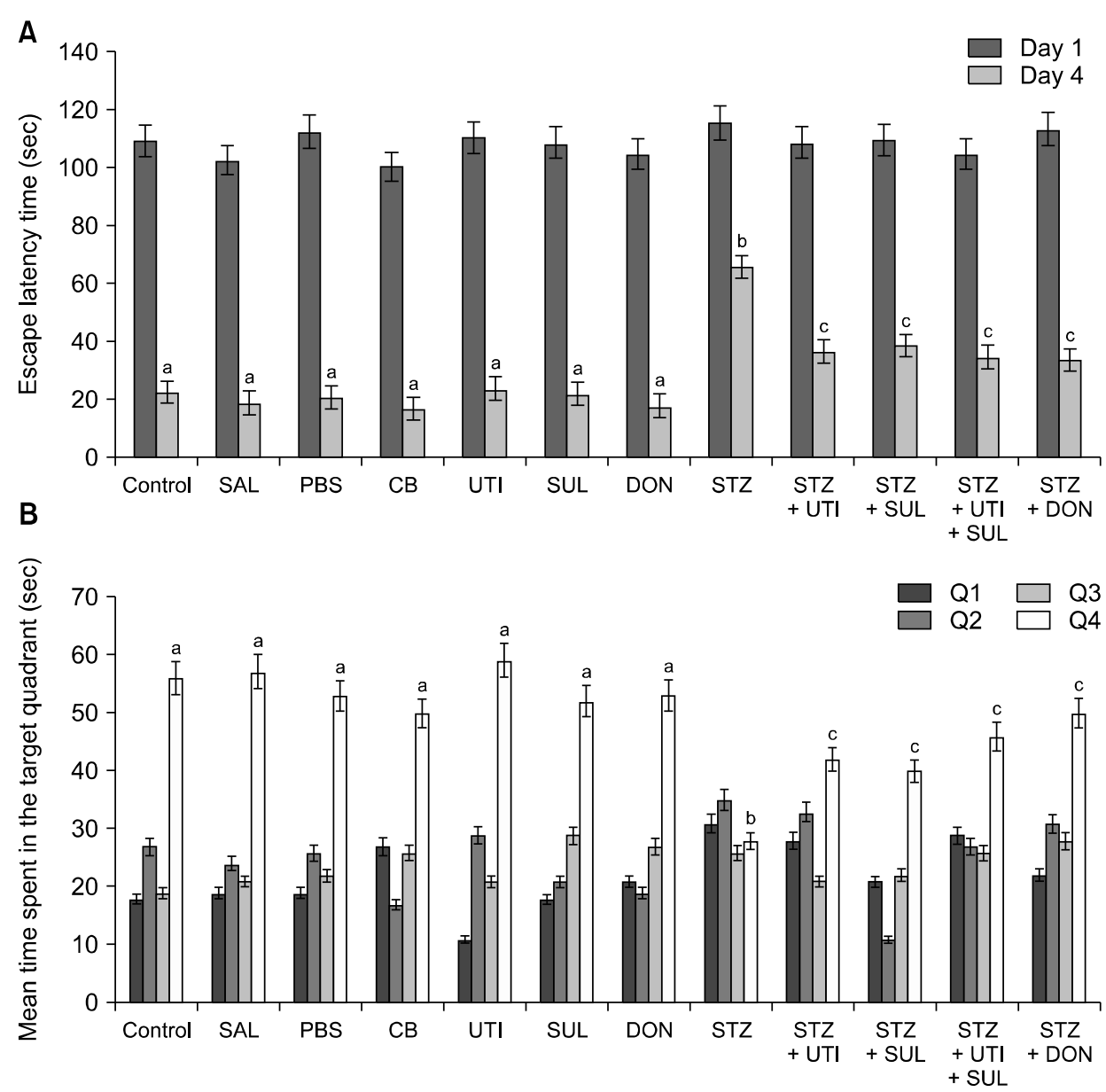

Fig. 1. Effect on learning and memory using MWM. Results are expressed as mean \pm standard deviation $(n=8)$, and were statistically analysed using one-way ANOVA (sigma stat 12.5) followed by Bonferroni's post hoc test. (A) ELT $=\mathrm{F}(11,84)=113.474 ;{ }^{\mathrm{a}} p<0.05$ vs. day 1 in respective group; ${ }^{\mathrm{b}} p<0.05$ vs. day 4 in control group. ${ }^{\mathrm{c}} p<0.05$ vs. day 4 in STZ group. (B) TSTQ $=\mathrm{F}(11,84)=47.124 ;{ }^{\mathrm{a}} p<0.05$ vs. mean time spent in other quadrant in respective group; ${ }^{\mathrm{b}} p<0.05$ vs. mean time spend in target quadrant by control group; ${ }^{\mathrm{c}} p<0.05 \mathrm{vs}$. mean time spend in target quadrant by STZ group.

MWM, Morris water maze; SAL, saline; PBS, phosphate-buffered saline; CB, citrate buffer; UTI, ulinastatin; SUL, sulforaphane; DON, donepezil; STZ, streptozotocin.

STZ diabetes induced reduction in the serum nitrite/nitrate levels $(\mathrm{F}[11,84]=46.991, p<0.05)$ as well as STZ diabetes induced increase in the aortic superoxide anion (F [11, 84], $p<0.05$ ) levels (Fig. 4A, B).

\section{Effect on Brain Oxidative Stress (TBARS, GSH, SOD, CAT)}

Compared to the control animals, STZ diabetic rats showed an increase in brain oxidative markers (TBARS) and decrease in brain antioxidant markers (GSH, SOD, CAT). Treatment with UTI (10,000 U/kg, i.p.) and SUL (25 $\mathrm{mg} / \mathrm{kg}$ ) alone as well as in combination and donepezil $(0.5 \mathrm{mg} / \mathrm{kg}$, i.p., daily) prevented STZ diabetes induced increase in the brain TBARS $(F[11,84]=124.657, p<$
0.05) levels as well as decrease in the brain's anti-oxidant markers that is., GSH $(\mathrm{F}[11,84]=54.356, p<0.05)$; $\operatorname{SOD}(\mathrm{F}[11,84]=71.315, p<0.05)$; $\mathrm{CAT}(\mathrm{F}[11,84]=$ 105.789, $p<0.05$ ) (Fig. 5A-D).

\section{Effect on Brain Inflammatory Markers (IL-6, IL-10, TNF- $\alpha$, MPO)}

Compared to the control animals, STZ diabetic rats showed an increase in brain inflammatory markers (IL-6, TNF- $\alpha$, MPO) and decrease in brain anti-inflammatory marker (IL-10). Treatment with UTI (10,000 U/kg, i.p.) and SUL $(25 \mathrm{mg} / \mathrm{kg}$ ) alone as well as in combination and donepezil $(0.5 \mathrm{mg} / \mathrm{kg}$, i.p., daily) prevented STZ diabetes induced increase in the brain IL-6 (F [11, 84] $=648.989, p<$ 


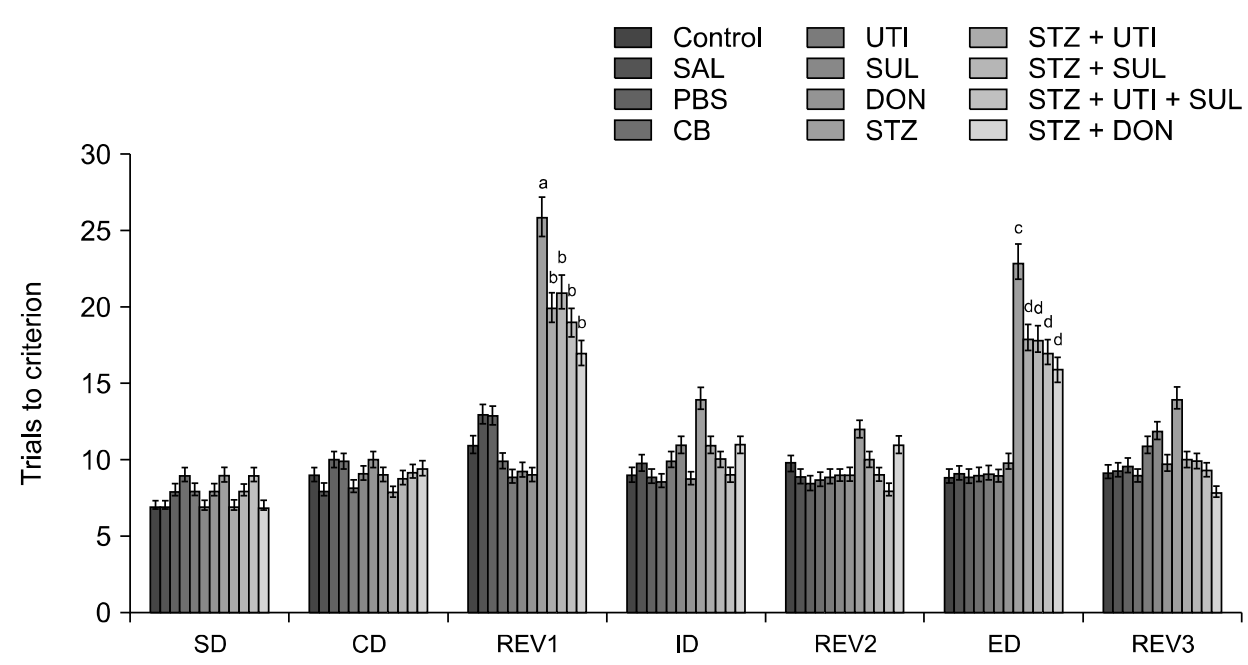

Fig. 2. Effect on reversal learning and executive functioning using ASST. Results are expressed as mean \pm standard deviation $(n=8)$, and were statistically analyzed using one-way ANOVA (sigma stat 12.5) followed by Bonferroni post hoc test.

REV $1=\mathrm{F}(11,84)=385.836 ;{ }^{\mathrm{a}} p<0.05$ vs. control group; ${ }^{\mathrm{b}} p<0.05$ vs. of STZ group. $\mathrm{ED}=\mathrm{F}(11,84)=442.116 ;{ }^{\mathrm{c}} p<0.05$ vs. control group; ${ }^{\mathrm{d}} p<$ 0.05 vs. STZ group.

ASST, attentional set shifting tests; SAL, saline; PBS, phosphate-buffered saline; CB, citrate buffer; UTI, ulinastatin; SUL, sulforaphane; DON, donepezil; STZ, streptozotocin.

0.05); TNF- $\alpha(\mathrm{F}[11,84]=214.838, p<0.05) ;$ and, $\mathrm{MPO}$ levels $(F[11,84]=242.955, p<0.05)$ as well as decrease in the brain IL-10 levels $(\mathrm{F}[11,84]=150.26, p<0.05)$ (Fig. 6A, B).

\section{Effect on Brain AChE Activity}

Compared to the control animals, STZ diabetic rats showed an increase in brain AChE activity. Treatment with UTI (10,000 U/kg, i.p.) and SUL (25 mg/kg) alone as well as in combination and donepezil $(0.5 \mathrm{mg} / \mathrm{kg}$, i.p., daily) prevented STZ diabetes induced increase in the brain AChE activity ( $[11,84]=302.228, p<0.05)$ (Fig. 7).

\section{Effect on BBB Permeability}

Compared to the control animals, STZ diabetic rats showed an increase in Evans blue dye extravasation in cerebral cortex depicting impairment in BBB permeability. Treatment with UTI (10,000 U/kg, i.p.) and SUL $(25 \mathrm{mg} / \mathrm{kg})$ alone as well as in combination and donepezil $(0.5 \mathrm{mg} / \mathrm{kg}$, i.p., daily) prevented STZ diabetes induced increase in Evans blue dye extravasation ( $F[11,84]=2,997.317, p<$ 0.05) (Fig. 8).

\section{Effect on Histopathological Studies}

STZ animals' images of Nissl staining on cerebral cortex depicts that STZ diabetic animals as compared to control group, have increased number of pyknotic and chromatolytic cells. Treatment with UTI $(10,000 \mathrm{U} / \mathrm{kg}$, i.p.) and SUL $(25 \mathrm{mg} / \mathrm{kg}$ ) alone as well as in combination and donepezil $(0.5 \mathrm{mg} / \mathrm{kg}$, i.p., daily) significantly attenuated the STZ diabetes induced increase in the number of pyknotic and chromatolytic cells in the cerebral cortex of the STZ diabetic rats $(F[4,35]=31.267, p<0.05)$ (Fig. 9).

\section{DISCUSSION}

In present investigation, STZ diabetes increased serum glucose levels (assessed every week), reduced body weight, learning, memory (MWM), reversal memory, executive functioning (ASST) and impaired endothelial function as well as BBB permeability in rats (assessed using thoracic aorta). STZ diabetic rats showed increased cerebral oxidative stress ( $\uparrow$ TBARS, $\downarrow$ GSH,$\downarrow$ SOD, $\downarrow$ CAT), inflammation ( $\uparrow$ IL-6, $\uparrow$ TNF- $\alpha, \uparrow$ MPO, $\downarrow$ IL-10), AChE activity, and histopathological changes. The results obtained are in accordance with the previously published result reports from ours as well as others' lab [8-11]. Further, treatment with UTI as well as SUL alone as well as in combination, attenuated the behavioral deficits, endothelial dysfunction, impaired biochemical parameters, and histopathological changes in STZ diabetic rats. 

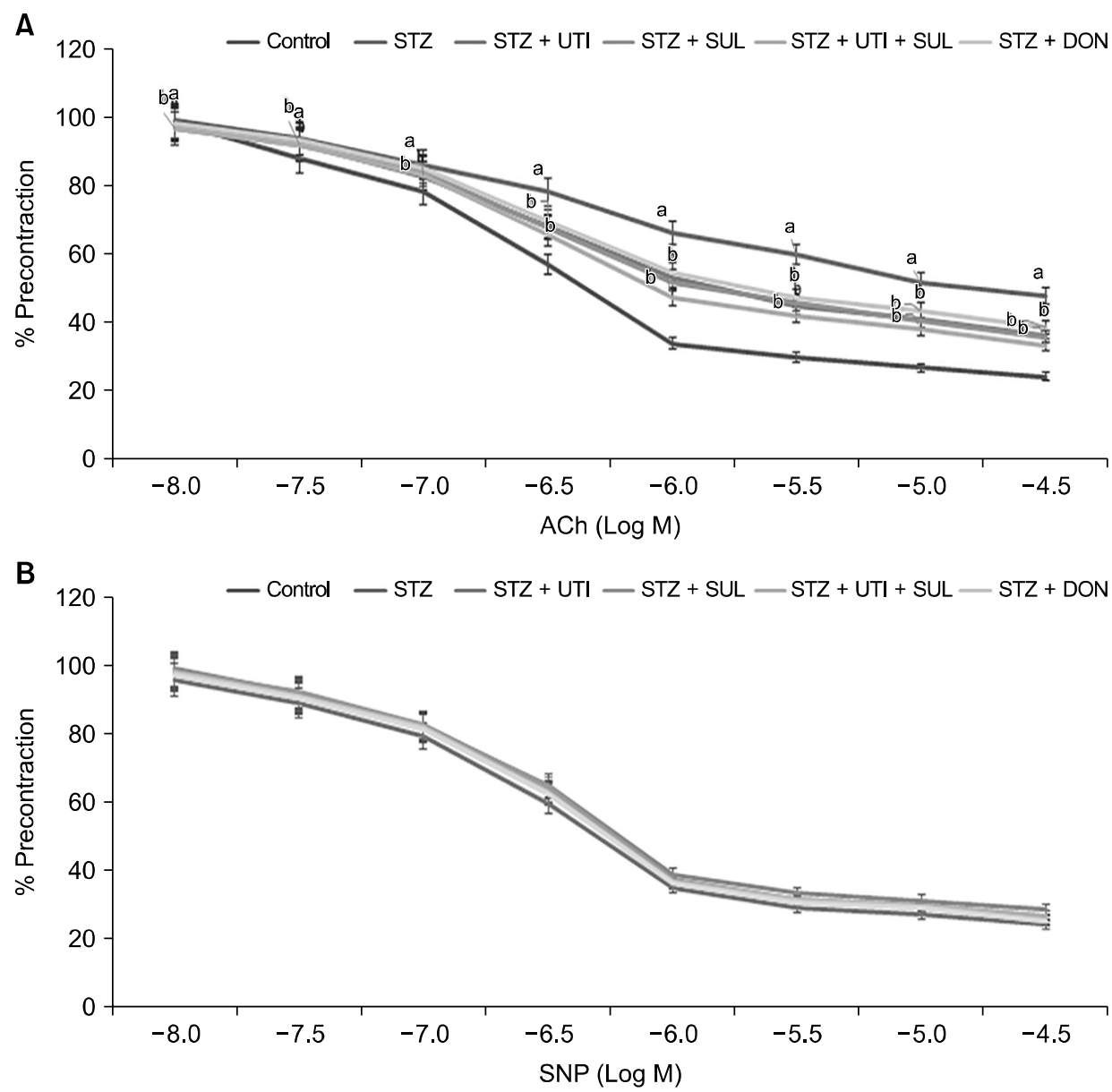

Fig. 3. Effect on endothelium dependent and independent relaxation. Results are expressed as mean \pm standard deviation $(n=8)$, repeated measure ANOVA (sigma stat 12.5) followed by Newman-Keul's test. (A) Endothelium dependent relaxation $={ }^{\mathrm{a}} p<0.05 \mathrm{vs}$. control group; ${ }^{\mathrm{b}} p<0.05 \mathrm{vs}$. STZ group. (B) Endothelium independent relaxation.

UTI, ulinastatin; SUL, sulforaphane; DON, donepezil; STZ, streptozotocin.

STZ diabetic rats shows loss in the body weight. This is probably due to their inability to metabolize carbohydrates which, shifts the reliance more towards fat metabolism for the energy purposes hence, results into wastage of fat resources [42]. Administration of STZ causes death of the insulin producing $\beta$ cells in the pancreas, which increases the serum glucose levels in rats [4]. Thus, we have utilized STZ for induction of diabetes. Diabetes inhibits long term potentiation in hippocampal cells. This is responsible for mammalian learning and memory by decreasing nitric oxide (NO) [43], which itself is associated with memory deficits [9-11,34,35]. Our previous studies in diabetic rats have reported reduction in learning and memory due to endothelial dysfunction, increased oxidative stress, inflammation, and AChE activity [9-11,34,39]. This work and our previous works utilized STZ-induced diabetes for induction of endothelial dysfunction, behavioral deficits, and biochemical impairments of vascular dementia [8-11]. Even other research groups have also assessed similar alterations induced by diabetes $[42,44,45]$. Thus, in view of the results of this study and results of previous studies both from our lab and other research groups $[4,8-11,34,42,45]$ we can say possibly it is the STZ diabetes which is responsible to induce changes in animals similar to that of phenotypes of vascular dementia. Therefore, in this study diabetes may have impaired learning, memory, reversal learning and executive functioning, via endothelial dysfunction, cholinergic dysfunction, oxidative stress and inflammation.

Endothelium maintains the balance between the vasoconstriction and vasodilation. Diabetes upregulates inducible nitric oxide synthase (iNOS) and impairs activa- 

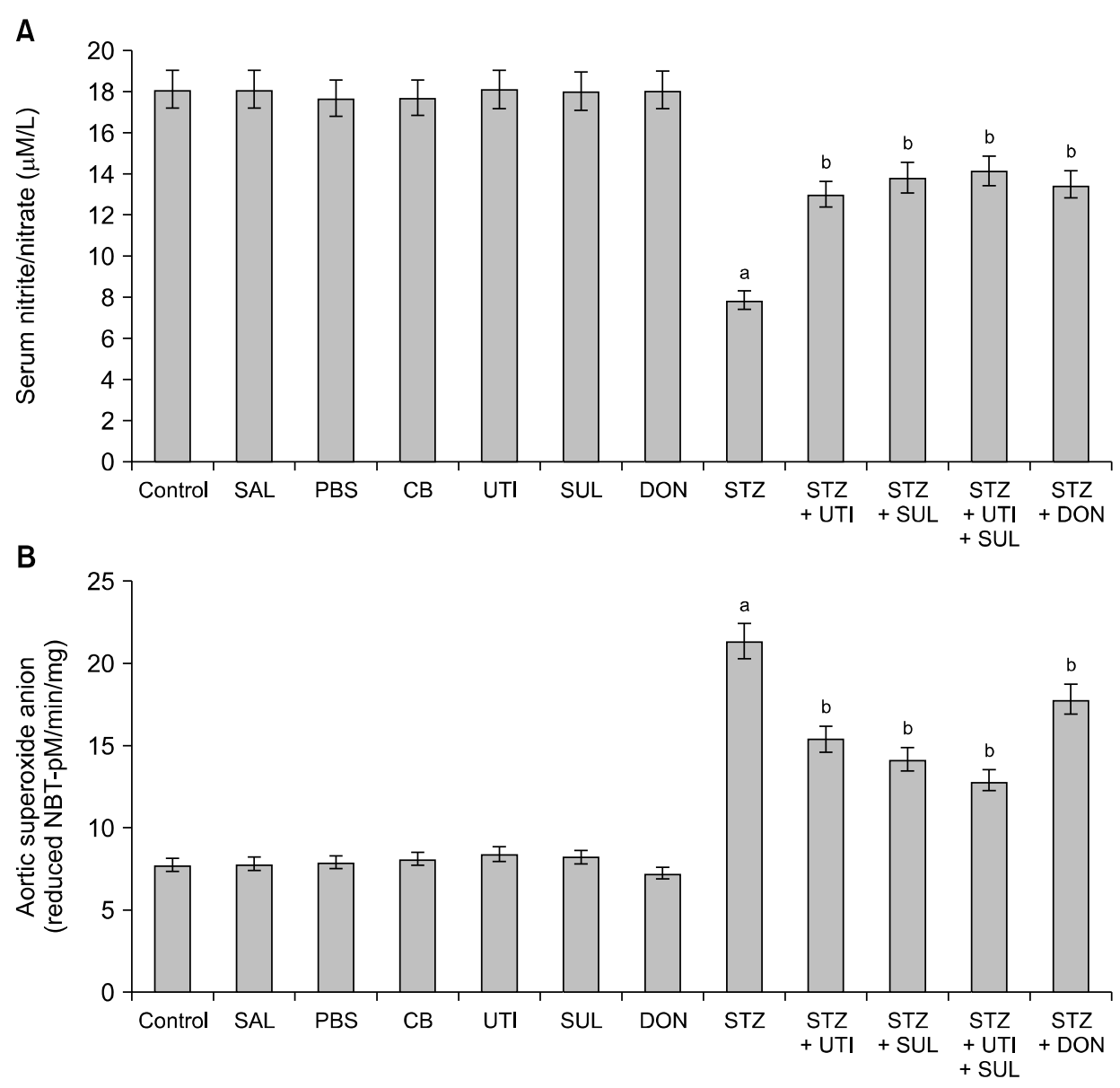

Fig. 4. Effect on serum nitrite/ nitrate and aortic superoxide anion. Results are expressed as mean \pm standard deviation $(n=8)$, one-way ANOVA (sigma stat 12.5) followed by Bonferroni's post hoc test. ${ }^{\mathrm{a}} p<0.05$ vs. control group; ${ }^{\mathrm{b}} p<0.05 \mathrm{vs}$. STZ group. (A) Serum nitrite/nitrate $=\mathrm{F}(11,84)=$ 46.991. (B) Aortic superoxide anion $=F(11,84)=220.936$.

SAL, saline; PBS, phosphate-buffered saline; CB, citrate buffer; UTI, ulinastatin; SUL, sulforaphane; DON, donepezil; STZ, streptozotocin.

tion of the endothelium nitric oxide synthase (eNOS) which, causes reduction in the serum nitrite/nitrate levels, as observed in the present as well as previous studies $[35,44]$. Hyperglycemia increases NOS mediated vascular superoxide production by increasing nicotinamide adenine dinucleotide phosphate hydrogen (NAD $[\mathrm{P}] \mathrm{H})$ oxidase activity in diabetic patients [46]. This causes endothelial dysfunction. Increase in the oxidative stress is another pathological feature of diabetes [46]. ROS causes lipid peroxidation by mainly targeting the polyunsaturated fatty acids on the cell membrane as observed by increased TBARS levels $[34,35,40]$. Excessive production of superoxide and hydroxyl levels, decreases the antioxidant SOD activity [47]. Diabetes mediated increase in NADH production, decreases $\mathrm{NAD}(\mathrm{P}) \mathrm{H}$ levels which is required for the formation of GSH [48] which, decreases the GSH levels. CAT act as a main regulator for the metabolism of hydrogen peroxide, consistent hyperglycemia downregulates the CAT protein expression $[49,50]$. Hyperglycemia increase brain MPO, TNF- $\alpha$ and IL-6 levels [51,52]. Hence, increase in brain TBARS, TNF- $\alpha$, IL-6, MPO as well as decrease in brain SOD, GSH, and CAT have been previously observed in STZ diabetic rats. Further, endothelial dysfunction, cholinergic dysfunction, inflammation and oxidative stress in STZ diabetes have resulted in cognitive dysfunction [7-9,39,40,52].

UTI administration reduces the STZ diabetes induced weight loss in the current study. UTI reduces the loss in weight possibly via inhibiting the hyperglycemia induced activation of the inflammatory and apoptotic pathway as observed in the clinical study as well as rodent study [53-55]. Clinical data suggests that UTI reduces hyperglycemia via improving the insulin resistance and reducing the excessive release of inflammatory factors [56]. 

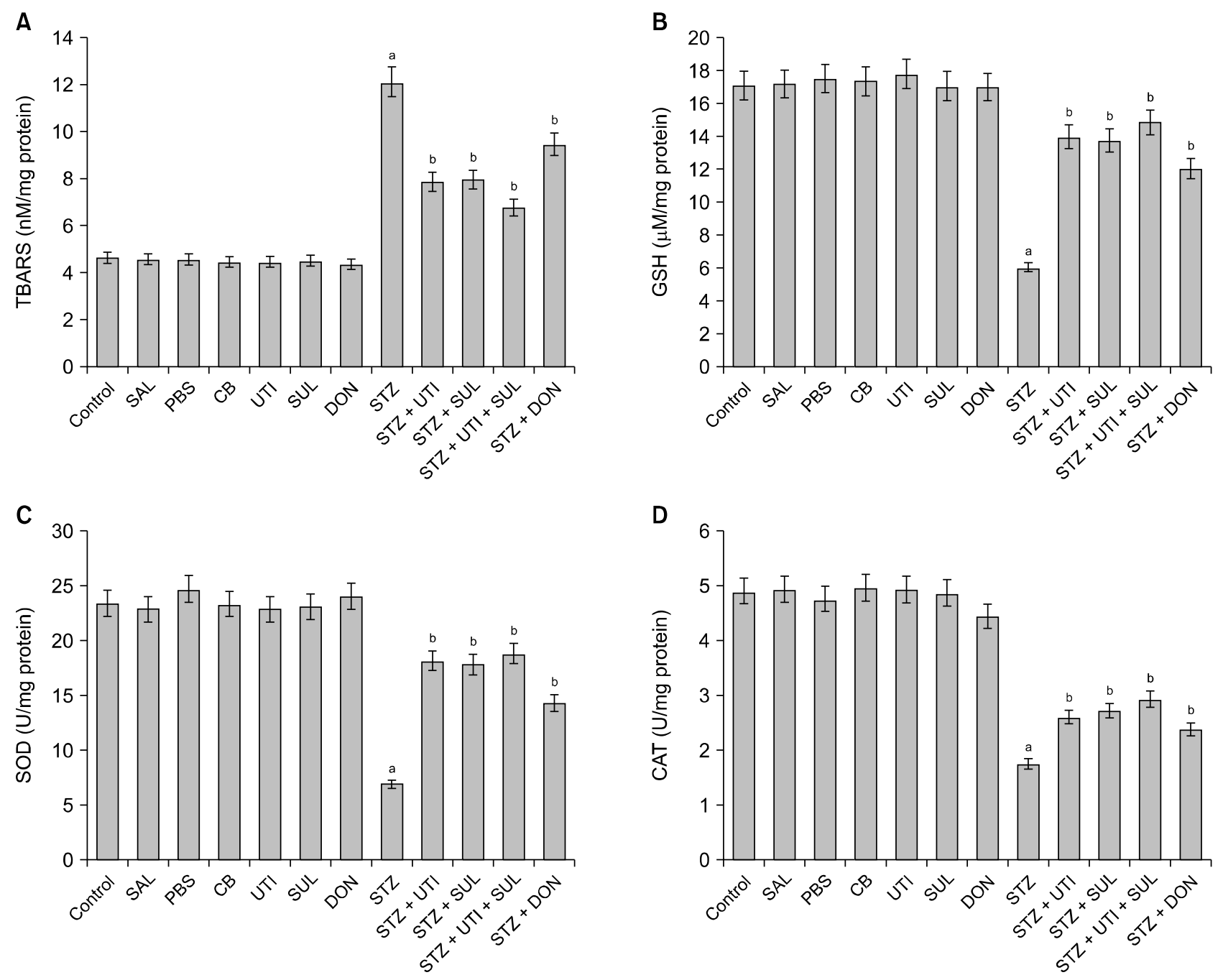

Fig. 5. Effect on brain oxidative stress (TBARS, GSH, SOD, CAT). Results are expressed as mean \pm standard deviation $(\mathrm{n}=8)$, one-way ANOVA (sigma stat 12.5 ) followed by Bonferroni's post hoc test. ${ }^{\mathrm{a}} p<0.05$ vs. control group; ${ }^{\mathrm{b}} p<0.05$ vs. STZ group. (A) TBARS $=\mathrm{F}(11,84)=124.657$. (B) $\mathrm{GSH}=\mathrm{F}(11,84)=54.356$. (C) $\mathrm{SOD}=\mathrm{F}(11,84)=71.315$. (D) $\mathrm{CAT}=\mathrm{F}(11,84)=105.789$.

SAL, saline; PBS, phosphate-buffered saline; CB, citrate buffer; UTI, ulinastatin; SUL, sulforaphane; DON, donepezil; STZ, streptozotocin.

Furthermore, UTI has shown benefits against impairment in learning, memory, spatial memory, attention and cognition during pre-clinical and clinical studies $[57,19]$. Beneficial and protective effects of UTI has been observed on synaptic plasticity [57]. UTI is reported to translocate lipopolysaccharide in patients undergoing spinal surgery [19]. Human UTI like substances are observed in the rat's hippocampus, cerebral cortex and hypothalamus region which are partly related to learning and memory $[58,59]$. In addition to this, UTI administration inhibits nuclear factor kappa-B (NF-kB) activation and hence inflammation [60]. Protective effects of UTI has also been observed against cholinergic dysfunction, oxidative stress, endo- thelial dysfunction, BBB disruption and neuronal apoptosis $[12,13,17,20,21]$. The increase in inflammation, oxidative stress, cholinergic dysfunction and endothelial dysfunction have already been reported to cause learning and memory impairment, in our previous studies $[18,34,35]$ Therefore, beneficial effects of UTI on spatial memory, learning, reversal memory and executive functioning, observed in this study may have been due to its beneficial effect against endothelial dysfunction, oxidative stress, inflammation, and AChE activity.

UTI administration in the current study increases the serum nitrite/nitrate levels. This is possibly due to UTI induced phosphorylation/activation of endothelial nitric oxide 

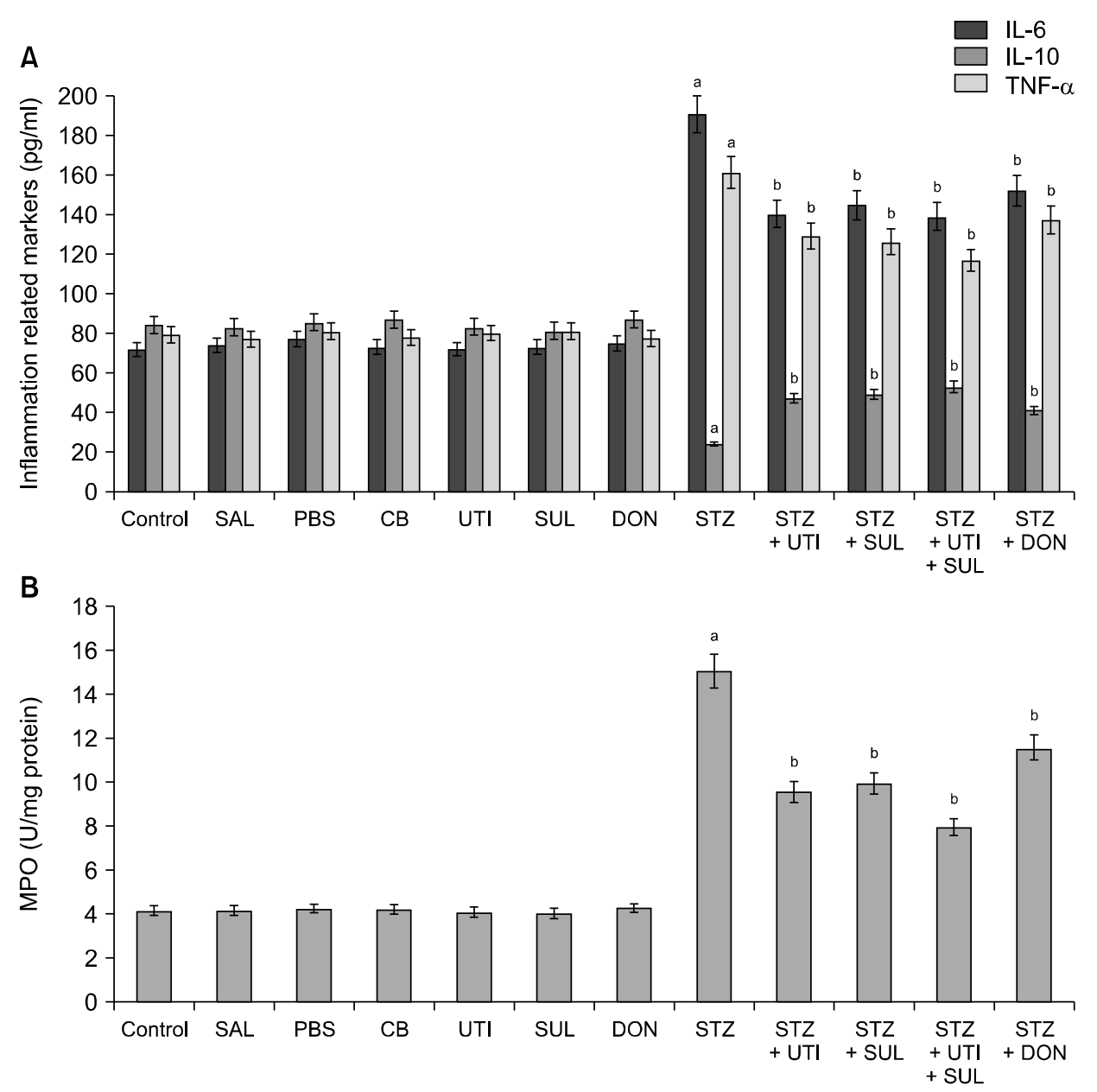

Fig. 6. Effect on brain inflammatory markers (IL-6, IL-10, TNF- $\alpha, M P O)$. Results are expressed as mean \pm standard deviation $(n=8)$, one-way ANOVA (sigma stat 12.5 ) followed by Bonferroni's post hoc test. ${ }^{\mathrm{a}} p<0.05$ vs. control group; ${ }^{\mathrm{b}} p<0.05$ vs. STZ group. (A) IL-6 $=\mathrm{F}(11,84)=$ 648.989. (A) IL-10 $=F(11,84)=150.26$. (A) TNF- $\alpha=F(11,84)=214.838$. (B) $M P O=F(11,84)=242.955$.

SAL, saline; PBS, phosphate-buffered saline; CB, citrate buffer; UTI, ulinastatin; SUL, sulforaphane; DON, donepezil; STZ, streptozotocin.

synthetase (eNOS) and AKT which, increases the levels of intracellular NO [61]. Also, UTI protects against vascular hyperpermeability by modulating the intrinsic apoptotic signaling pathway [62]. Furthermore, UTI improves permeability and integrity of the BBB by inhibiting the expression of matrix metallopeptidase 9 (MMP-9) and tissue plasminogen activator (t-PA) [14]. Furthermore, UTI inhibits the reduced nicotinamide adenine dinucleotide phosphate (NADPH) oxidase and NF-kB which, further inhibits vascular superoxide production and inflammation (TNF- $\alpha$, interleukin-1 $\beta$ (IL-1 $\beta$ ), IL-6, and MPO) $[60,63]$. UTI reduces the lipid peroxidation as observed by the reduced TBARS levels [64]. UTI mediated increase in the anti-oxidants (SOD, GSH, CAT) is possibly via upregulation of nuclear factor E2-related factor 2 (Nrf2) gene
$[21,22,65]$ and activation of Nrf2/NOS pathway [66]. Also, UTI administration regulates AMPK/NF-kB pathway, and downregulates (toll like receptor) TLR 4 which are further reported to decreases inflammation [67]. Hence, UTI shows beneficial effects against reduced learning, memory, endothelial dysfunction, oxidative stress, inflammation, and AChE activity which, may be responsible for its effects against diabetes induced vascular endothelial dysfunction and related dementia.

SUL administration in the current study reduces STZ diabetes induced decrease in the weight reduction possibly via reducing inflammation and oxidative stress [26]. SUL mediated upregulation of the Nrf2 gene is found responsible for its effect against hyperglycemia [26]. Furthermore, SUL has shown benefits against impairment of 


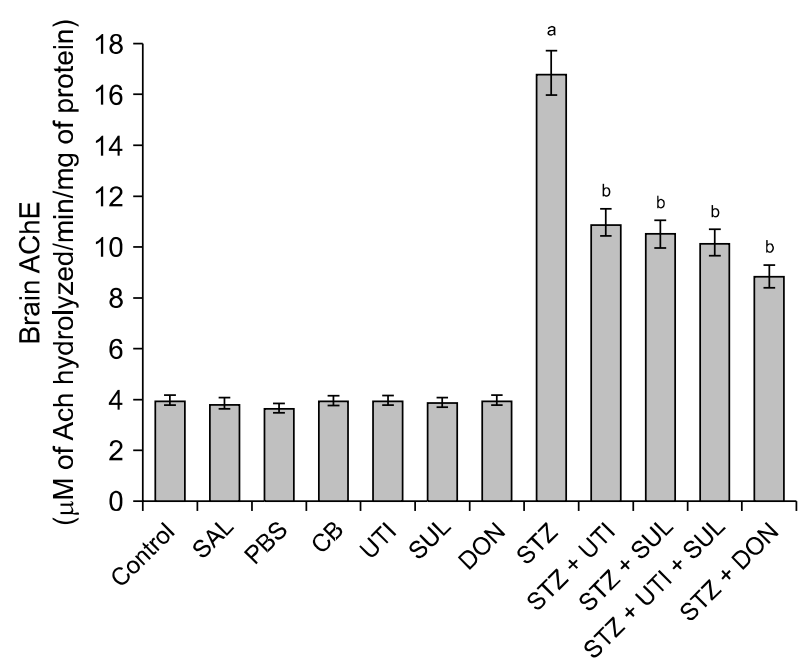

Fig. 7. Effect on brain AChE activity. Results are expressed as mean \pm standard deviation $(\mathrm{n}=8)$, one-way ANOVA (sigma stat 12.5) followed by Bonferroni's post hoc test. $\mathrm{F}(11,84)=302.228$. ${ }^{\mathrm{a}} p<0.05$ vs. control group; ${ }^{\mathrm{b}} p<0.05$ vs. STZ group.

SAL, saline; PBS, phosphate-buffered saline; CB, citrate buffer; UTI, ulinastatin; SUL, sulforaphane; DON, donepezil; STZ, streptozotocin.

learning, memory, cognition via inhibition of AChE activity [68] and activation of brain-derived neurotrophic factor (BDNF)-mTOR [27] as well as Nrf2-HO-1 (Heme oxygenase) signaling pathway [69]. SUL increases the expression of choline acetyltransferase (chAT) [68], Nrf2, and tissue inhibitor of metaloproteinases 2 (TIMP2) gene [70] that, promote learning, memory, spatial memory, synaptic plasticity and hippocampal function. Also, SUL prevents neuronal apoptosis of the hippocampal region via upregulating $\mathrm{p}$-Akt, phospho-glycogen synthase kinase 3 beta (p-GSK3 $\beta$ ), nerve growth factor (NGF) and BDNF expressions [33]. In addition to this, SUL administration modulates Nrf2 and inhibits NF-kB activation and therefore, production of inflammatory markers hence, inflammation [71,31]. Protective effects of SUL have also been observed against cholinergic dysfunction [68], oxidative stress [30], endothelial dysfunction, BBB disruption [28] and neuronal apoptosis [32]. Therefore, beneficial effects of UTI on spatial memory, learning, reversal memory and executive functioning, observed in this study may have been due to its beneficial effect against endothelial dysfunction, oxidative stress, inflammation, and AChE activity.

SUL administration has protective effect on the endothelial function and integrity of the BBB [28]. SUL administration in the current study increases the serum nitrite/ni-

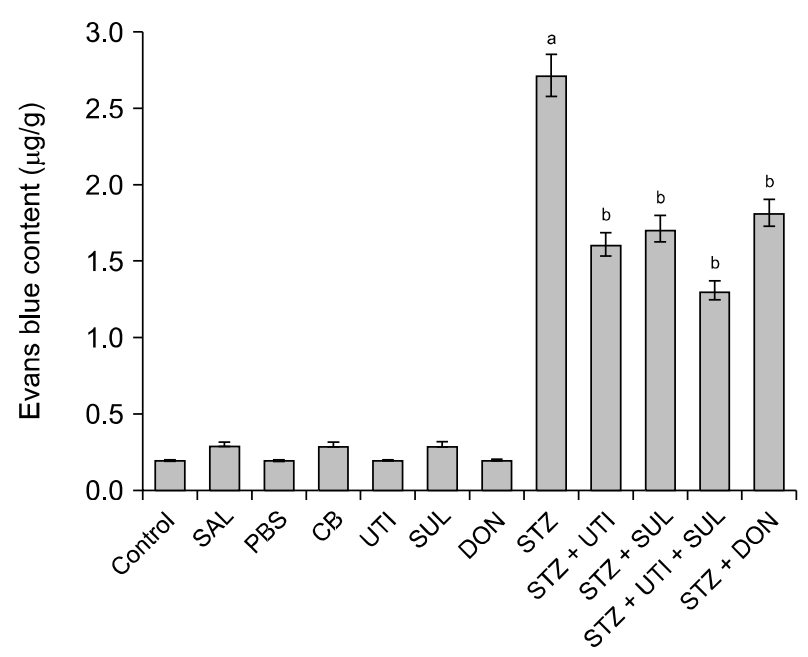

Fig. 8. Effect on BBB permeability. Results are expressed as mean \pm standard deviation $(n=8)$, one-way ANOVA (sigma stat 12.5$)$ followed by Bonferroni's post hoc test. $\mathrm{F}(11,84)=2997.317$. ${ }^{\mathrm{a}} p<0.05$ vs. control group; ${ }^{\mathrm{b}} p<0.05$ vs. STZ group.

BBB, blood brain barrier; SAL, saline; PBS, phosphate-buffered saline; $\mathrm{CB}$, citrate buffer; UTI, ulinastatin; SUL, sulforaphane; DON, donepezil; STZ, streptozotocin.

trate levels by increasing the expression of eNOS mRNA which, further increases the nitric oxide levels [72]. Also, in-vitro study suggests that, SUL administration inhibits the AGE-RAGE axis whose, excessive production are known to cause endothelial dysfunction [73]. In addition to this, SUL restores the BBB integrity via inhibiting MMP-9 expression and restoring claudin-5 as well as occluding expression [74]. The cumulative effect of all these may enhance the endothelial function. The activation of Nrf2 gene via SUL administration further, reduces the vascular superoxide production [72]. In addition to this, treatment with SUL reduces the lipid peroxidation as observed by the reduced TBARS levels in the present study also [30]. Furthermore, SUL mediates transcriptional activation of the Nrf2 gene with the antioxidant response element (ARE) in their promoters [75]. Nrf2/ARE activation by SUL further induces antioxidant enzymes (SOD, CAT, GSH) that enhance the antioxidative effect [76]. Furthermore, SUL decreases the levels of TNF- $\alpha$, IL-6, MPO and increases the levels of IL-10 by inhibiting the NF-kB and activating the Nrf2 pathway [29]. Hence, SUL shows beneficial effects against reduced learning, memory, endothelial dysfunction, oxidative stress, inflammation, and AChE activity which, may be responsible for its effects against diabetes induced vascular endothelial dysfunction and related dementia. 

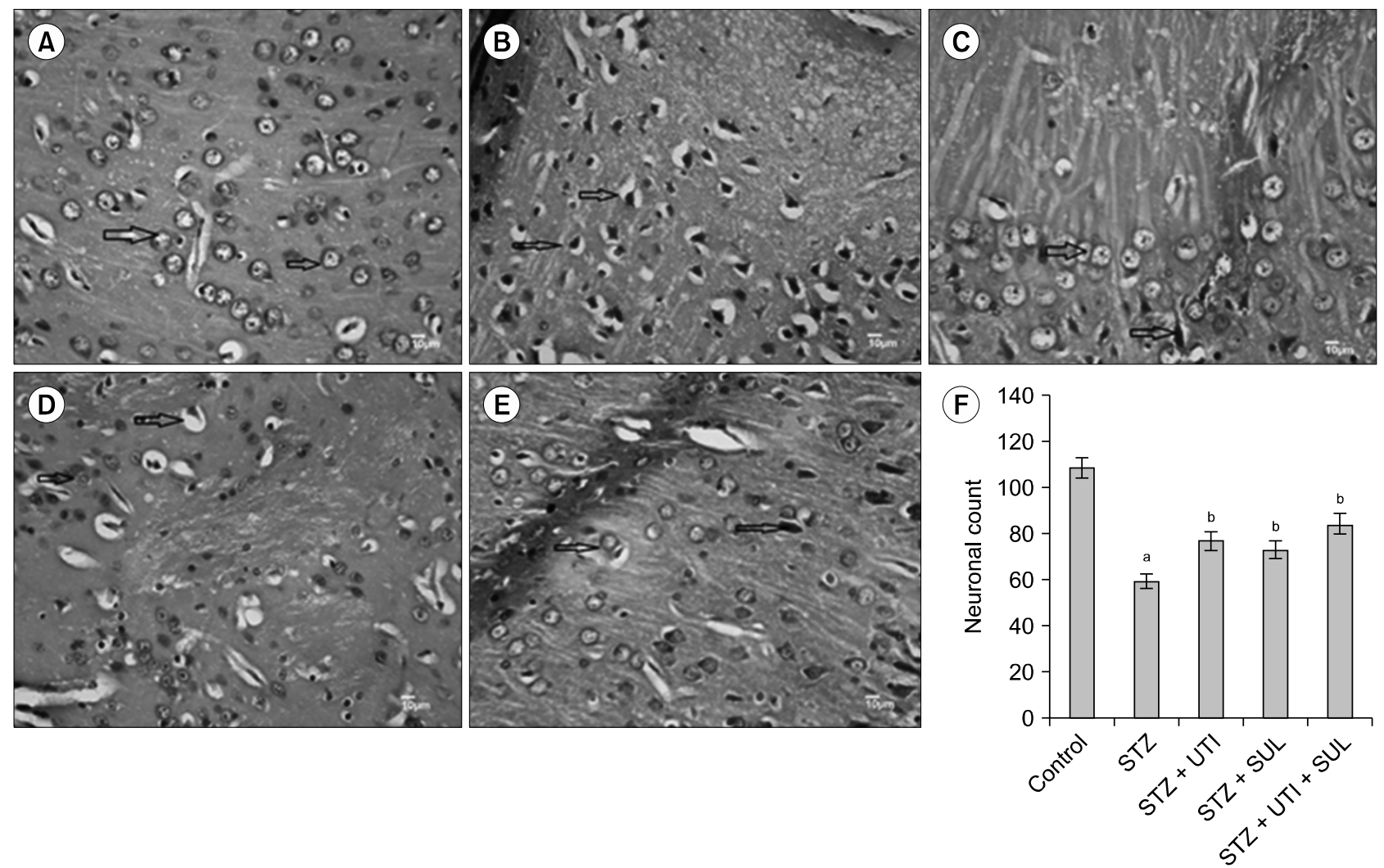

Fig. 9. Effect on histopathological studies. The Nissl staining of cerebral cortex of STZ diabetic rats (400× magnification; scale bar $=10 \mu \mathrm{m})$. (A) Control group, showing closely packed neuronal cells with vesicular nuclei; (B) STZ group, showing many pyknotic and chromatolytic cells with irregular shape and condensed nuclei; (C) STZ + UTI group, showing few closely packed neuronal cells along with less number of pyknotic and chromatolytic cells; (D) STX + SUL group, showing increased number of normal neuronal cells with fewer pyknotic and chromatolytic cells; (E) STZ + UTI + SUL group, showing greater number of normal closely packed neuronal cells along with very few number of pyknotic cells depicting beneficial effect of combination; (F) neuronal count. ${ }^{a} p<0.05$ vs. control group; ${ }^{b} p<0.05$ vs. STZ group.

Results are expressed as mean \pm standard deviation $(n=8)$, one-way ANOVA (sigma stat 12.5$)$ followed by Bonferroni's post hoc test. $F(4,35)=$ 31.267.

UTI, ulinastatin; SUL, sulforaphane; STZ, streptozotocin.

Furthermore, as UTI is known to inhibit neuronal apoptosis [21,55,62], cognitive dysfunction [16], cerebral edema [14], oxidative stress [21,55], inflammation $[15,16]$, as well as cerebral expression of MMP-9, Zonula occludens-1 (ZO-1), occludin [15], and $\beta$-amyloid proteins [16], the beneficial effects of UTI in the current study may have been due to its neuroprotective effect. Also, SUL is known to ameliorate vascular cognitive impairment [27], neuronal apoptosis [33], neurotoxicity [33], oxidative stress [30], neuroinflammation [29,31], depression like behavior, and promote neurogenesis [24] therefore, the beneficial effects of SUL in the present study may have been due to its neuroprotective effects. Also, On the basis of our data, we observed that, the combination therapy of UTI and SUL provide better neuroprotection against diabetes induced endothelial dysfunction and related de- mentia, as compared to neuroprotection observed by the administration of UTI and SUL alone. Furthermore, it is important to mention that, this is the first study which is assessing the combinatorial effect of these agents. No other study has previously assessed the combinational effect of these agents. UTI and SUL both activates Nrf2 and inhibits NF-kB pathway $[31,60,71]$. SUL further activates KEAP1 independent of Nrf2 that increases the expression of anti-oxidant enzymes [76]. Therefore, the reduction in the oxidative stress and inflammation using combinatorial therapy could have been due to the synergistic aspect of the drug combination.

Donepezil, a highly selective, potent, and reversible inhibitor of the AChE is a well-established drug for the management of memory dysfunction in AD patients [77]. Clinical study has already demonstrated the benefits of 
donepezil in inflammation, oxidative stress, and impaired cognition [77]. Further, its efficacy has already been demonstrated in several experimental models of $\mathrm{VaD}$ [8-11]. Hence, we have selected donepezil and used it as a positive control in the present study.

Thus, we can conclude that, UTI and SUL alone as well as in combination, provide beneficial effects by improving learning, memory, reversal memory, executive functioning, endothelial function, oxidative status, inflammation, and brain cholinergic activity in STZ induced diabetes induced endothelial dysfunction and related $\mathrm{VaD}$. However, the anti-diabetic effect and dose dependent effect of the target drugs, were not studied in the present study. Also, the elaborative mechanistic study of the target drugs in neuroprotection were not assessed which, becomes the limitation of this study. Thus, future studies should be planned to assess the dose- dependent and anti-diabetic effects of these agents along with, identifying elaborative mechanisms to elucidate the full potential of these agents in diabetes induced vascular endothelial dysfunction and related dementia.

\section{Acknowledgments}

Authors are thankful to Dr. Ashok K. Chauhan, Hon'ble Founder President, Ritnand Balved Education Foundation, India and Dr Atul Chauhan, Hon'ble Chancellor, Amity University Uttar Pradesh, India, for equipping us with all the required experimental facilities and immense motivation towards the conduct this research work. We, also thank Prof. (Dr.) Nirmal Singh, Pharmacology Division, Department of Pharmaceutical Sciences and Drug Research, Faculty of Medicine, Punjab University, Patiala (Punjab), India, for his valuable suggestions.

\section{Conflicts of Interest}

No potential conflict of interest relevant to this article was reported.

\section{- Author Contributions}

Carried out the experiments, wrote the first draft of manuscript: Prachi Kaushik, Poonam Sharma, and Swati Jain. Conceived and planned the experiments: Brij Mohan Sharma and Bhupesh Sharma. Interpretation of results: Brij Mohan Sharma, Rajendra Awasthi, and Bhupesh Sharma. Encouraged, reviewed and edited manuscript: Giriraj Thirupathirao Kulkarni and Bhupesh Sharma. Supervised, analysed and discussed the subsequent drafts of manuscript: Bhupesh Sharma. All the authors approved the final manuscript.

\section{ORCID}

Poonam Sharma https://orcid.org/0000-0001-6271-8374

Prachi Kaushik https://orcid.org/0000-0003-3487-8543

Swati Jain https://orcid.org/0000-0002-2225-2650

Brij Mohan Sharma https://orcid.org/0000-0001-6942-263X

Rajendra Awasthi https://orcid.org/0000-0002-1286-1874

Giriraj Thirupathirao Kulkarni

https://orcid.org/0000-0002-9563-9469

Bhupesh Sharma https://orcid.org/0000-0002-3423-007X

\section{REFERENCES}

1. O’Brien JT, Thomas A. Vascular dementia. Lancet 2015;386: 1698-1706.

2. Wang J, Zhang HY, Tang XC. Cholinergic deficiency involved in vascular dementia: possible mechanism and strategy of treatment. Acta Pharmacol Sin 2009;30:879-888.

3. de la Monte SM, Wands JR. Alzheimer's disease is type 3 diabetes-evidence reviewed. J Diabetes Sci Technol 2008;2. 1101-1113.

4. Ravona-Springer R, Schnaider-Beeri M. The association of diabetes and dementia and possible implications for nondiabetic populations. Expert Rev Neurother 2011;11:16091617.

5. Prasad S, Sajja RK, Naik P, Cucullo L. Diabetes mellitus and blood-brain barrier dysfunction: an overview. J Pharmacovigil 2014;2:125.

6. Arnold SE, Arvanitakis Z, Macauley-Rambach SL, Koenig AM, Wang HY, Ahima RS, et al. Brain insulin resistance in type 2 diabetes and Alzheimer disease: concepts and conundrums. Nat Rev Neurol 2018;14:168-181.

7. Murtishaw AS, Heaney CF, Bolton MM, Belmonte KCD, Langhardt MA, Kinney JW. Intermittent streptozotocin administration induces behavioral and pathological features relevant to Alzheimer's disease and vascular dementia. Neuropharmacology 2018;137:164-177.

8. Sharma B, Singh N. Pitavastatin and 4'-hydroxy-3'-methoxyacetophenone (HMAP) reduce cognitive dysfunction in vascular dementia during experimental diabetes. Curr NeurovasC Res 2010;7:180-191.

9. Sharma B, Singh N. Attenuation of vascular dementia by sodium butyrate in streptozotocin diabetic rats. Psychopharmacology (Berl) 2011;215:677-687.

10. Sharma B, Singh N. Behavioral and biochemical investigations to explore pharmacological potential of PPAR-gamma agonists in vascular dementia of diabetic rats. Pharmacol Biochem Behav 2011;100:320-329.

11. Singh G, Sharma B, Jaggi AS, Singh N. Efficacy of bosentan, a 
dual ETA and ETB endothelin receptor antagonist, in experimental diabetes induced vascular endothelial dysfunction and associated dementia in rats. Pharmacol Biochem Behav 2014; 124:27-35.

12. Liu M, Shen J, Zou F, Zhao Y, Li B, Fan M. Effect of ulinastatin on the permeability of the blood-brain barrier on rats with global cerebral ischemia/reperfusion injury as assessed by MRI. Biomed Pharmacother 2017;85:412-417.

13. Ma L, Zhang H, Liu YZ, Yin YL, Ma YQ, Zhang SS. Ulinastatin decreases permeability of blood--brain barrier by inhibiting expression of MMP-9 and $t$-PA in postoperative aged rats. Int J Neurosci 2016;126:463-468.

14. Cui T, Zhu G. Ulinastatin attenuates brain edema after traumatic brain injury in rats. Cell Biochem Biophys 2015;71. 595-600.

15. Li XF, Zhang XJ, Zhang C, Wang LN, Li YR, Zhang Y, et al. Ulinastatin protects brain against cerebral ischemia/reperfusion injury through inhibiting MMP-9 and alleviating loss of ZO-1 and occludin proteins in mice. Exp Neurol 2018;302: 68-74.

16. Guo M, Zhu X, Xu H, Li J, Yang S, Zuo Z, et al. Ulinastatin attenuates isoflurane-induced cognitive dysfunction in aged rats by inhibiting neuroinflammation and $\beta$-amyloid peptide expression in the brain. Neurol Res 2019;41:923-929.

17. Inagaki T, Shikimi $T$, Fujimoto A, Ishino $H$, Okunishi $H$, Takaori S. Changes in the ratio of urinary alpha 1-microglobulin to ulinastatin levels in patients with psychiatric diseases. Biol Psychiatry 1996;40:800-803.

18. Cao LJ, Wang J, Hao PP, Sun CL, Chen YG. Effects of ulinastatin, a urinary trypsin inhibitor, on synaptic plasticity and spatial memory in a rat model of cerebral ischemia/reperfusion injury. Chin J Physiol 2011;54:435-442.

19. Zhang M, Zhang YH, Fu HQ, Zhang QM, Wang TL. Ulinastatin may significantly improve postoperative cognitive function of elderly patients undergoing spinal surgery by reducing the translocation of lipopolysaccharide and systemic inflammation. Front Pharmacol 2018;9:1007.

20. Koga Y, Fujita M, Tsuruta R, Koda Y, Nakahara T, Yagi T, et al. Urinary trypsin inhibitor suppresses excessive superoxide anion radical generation in blood, oxidative stress, early inflammation, and endothelial injury in forebrain ischemia/reperfusion rats. Neurol Res 2010;32:925-932.

21. Hu CL, Xia JM, Cai J, Li X, Liao XX, Li H, et al. Ulinastatin attenuates oxidation, inflammation and neural apoptosis in the cerebral cortex of adult rats with ventricular fibrillation after cardiopulmonary resuscitation. Clinics (Sao Paulo) 2013;68: 1231-1238.

22. Xie X, Li T, Yuan H. Protective effects of Ulinastatin on oxidative stress and inflammation of rat-derived cardiomyocytes H9c2. Am J Trans/ Res 2019;11:7094-7103.

23. Jazwa A, Rojo Al, Innamorato NG, Hesse M, Fernández-Ruiz J, Cuadrado A. Pharmacological targeting of the transcription factor Nrf2 at the basal ganglia provides disease modifying therapy for experimental parkinsonism. Antioxid Redox Signal 2011;14:2347-2360.

24. Huang C, Wu J, Chen D, Jin J, Wu Y, Chen Z. Effects of sulforaphane in the central nervous system. Eur J Pharmacol 2019; 853:153-168.

25. Shiina A, Kanahara N, Sasaki T, Oda Y, Hashimoto T, Hasegawa $\mathrm{T}$, et al. An open study of sulforaphane-rich broccoli sprout extract in patients with schizophrenia. Clin Psychopharmacol Neurosci 2015;13:62-67.

26. Axelsson AS, Tubbs E, Mecham B, Chacko S, Nenonen HA, Tang Y, et al. Sulforaphane reduces hepatic glucose production and improves g/ucose control in patients with type 2 diabetes. Sci Trans/ Med 2017;9:eaah4477.

27. Gao J, Xiong B, Zhang B, Li S, Huang N, Zhan G, et al. Sulforaphane alleviates lipopolysaccharide-induced spatial learning and memory dysfunction in mice: the role of BDNFmTOR signaling pathway. Neuroscience. 2018;388:357-366.

28. Ibbotson K, Yell J, Ronaldson PT. Nrf2 signaling increases expression of ATP-binding cassette subfamily C mRNA transcripts at the blood-brain barrier following hypoxia-reoxygenation stress. Fluids Barriers CNS 2017;14:6.

29. Jang M, Cho IH. Sulforaphane ameliorates 3-nitropropionic acid-induced striatal toxicity by activating the Keap1-Nrf2ARE pathway and inhibiting the MAPKS and NF-KB pathways. Mol Neurobiol 2016;53:2619-2635.

30. Zhou Q, Chen B, Wang X, Wu L, Yang Y, Cheng X, et al. Sulforaphane protects against rotenone-induced neurotoxicity in vivo: Involvement of the mTOR, Nrf2, and autophagy pathways. Sci Rep 2016;6:32206.

31. Townsend BE, Johnson RW. Sulforaphane induces Nrf2 target genes and attenuates inflammatory gene expression in microglia from brain of young adult and aged mice. Exp Gerontol 2016;73:42-48.

32. Lee S, Kim J, Seo SG, Choi BR, Han JS, Lee KW, et al. Sulforaphane alleviates scopolamine-induced memory impairment in mice. Pharmacol Res 2014;85:23-32.

33. Wang G, Fang $\mathrm{H}$, Zhen $\mathrm{Y}, \mathrm{Xu} \mathrm{G}$, Tian J, Zhang $\mathrm{Y}$, et al. Sulforaphane prevents neuronal apoptosis and memory impairment in diabetic rats. Cell Physiol Biochem 2016;39:901907.

34. Jain S, Sharma B. Effect of ruthenium red, a ryanodine receptor antagonist in experimental diabetes induced vascular endothelial dysfunction and associated dementia in rats. Physiol Behav 2016;164(Pt A):140-150.

35. Jain S, Sharma B. Neuroprotective effect of selective DPP-4 inhibitor in experimental vascular dementia. Physiol Behav 2015;152(Pt A):182-193.

36. Sharma P, Kulkarni GT, Sharma B. Possible involvement of D2/D3 receptor activation in ischemic preconditioning mediated protection of the brain. Brain Res 2020;1748:147116.

37. Reddy SS, Shruthi K, Prabhakar YK, Sailaja G, Reddy GB. Implication of altered ubiquitin-proteasome system and ER stress in the muscle atrophy of diabetic rats. Arch Biochem 
Biophys 2018;639:16-25.

38. Tekula S, Khurana A, Anchi P, Godugu C. Withaferin- $A$ attenuates multiple low doses of Streptozotocin (MLD-STZ) induced type 1 diabetes. Biomed Pharmacother 2018;106: 1428-1440.

39. Gupta S, Sharma B. Pharmacological benefits of agomelatine and vanillin in experimental model of Huntington's disease. Pharmacol Biochem Behav 2014;122:122-135.

40. Mirza R, Sharma B. Beneficial effects of pioglitazone, a selective peroxisome proliferator-activated receptor- $\gamma$ agonist in prenatal valproic acid-induced behavioral and biochemical autistic like features in Wistar rats. Int I Dev Neurosci 2019;76:6-16.

41. Matsumura $\mathrm{Y}$, Kuro $T$, Kobayashi $\mathrm{Y}$, Konishi F, Takaoka M, Wessale JL, et al. Exaggerated vascular and renal pathology in endothelin-B receptor-deficient rats with deoxycorticosterone acetate-salt hypertension. Circulation 2000;102:27652773.

42. Howarth FC, Jacobson M, Shafiullah M, Adeghate E. Long-term effects of streptozotocin-induced diabetes on the electrocardiogram, physical activity and body temperature in rats. Exp Physiol 2005:90:827-835.

43. Kennedy DO. $B$ Vitamins and the brain: mechanisms, dose and efficacy--a review. Nutrients 2016;8:68.

44. Natali A, Toschi E, Baldeweg S, Ciociaro D, Favilla S, Saccà L, et al. Clustering of insulin resistance with vascular dysfunction and low-grade inflammation in type 2 diabetes. Diabetes 2006; 55:1133-1140.

45. Safavi M, Hosseini-Sharifabad A, Seyed-Yousefi Y, Rabbani M. Protective effects of citicoline and benfotiamine each alone and in combination on streptozotocin-induced memory impairment in mice. Clin Psychopharmacol Neurosci 2020; 18:81-92.

46. Guzik TJ, Mussa S, Gastaldi D, Sadowski J, Ratnatunga C, Pillai R, et al. Mechanisms of increased vascular superoxide production in human diabetes mellitus: role of $\mathrm{NAD}(\mathrm{P}) \mathrm{H}$ oxidase and endothelial nitric oxide synthase. Circulation 2002; 105:1656-1662.

47. Ighodaro OM, Akinloye OA. First line defence antioxidantssuperoxide dismutase (SOD), catalase (CAT) and glutathione peroxidase (GPX): Their fundamental role in the entire antioxidant defence grid. Alex J Med 2018;54:287-293.

48. Muriach M, Flores-Bellver M, Romero FJ, Barcia JM. Diabetes and the brain: oxidative stress, inflammation, and autophagy. Oxid Med Cell Longev 2014;2014:102158.

49. Armogida M, Spalloni A, Amantea D, Nutini M, Petrelli F, Longone $\mathrm{P}$, et al. The protective role of catalase against cerebral ischemia in vitro and in vivo. Int I Immunopathol Pharmacol 2011;24:735-747.

50. Patel H, Chen J, Das KC, Kavdia M. Hyperglycemia induces differential change in oxidative stress at gene expression and functional levels in HUVEC and HMVEC. Cardiovasc Diabetol 2013;12:142.
51. Hop HT, Reyes AWB, Huy TXN, Arayan LT, Min W, Lee HJ, et al. Activation of NF-KB-mediated TNF-induced antimicrobial immunity is required for the efficient Brucella abortus clearance in RAW 264.7 cells. Front Cell Infect Microbiol 2017;7: 437.

52. Catrysse L, van Loo G. Inflammation and the metabolic syndrome: the tissue-specific functions of NF-кB. Trends Cell Biol 2017:27:417-429.

53. Zhao G, Zhu Y, Yu D, Ma J. The effect of ulinastatin on hyperglycemia in patients undergoing hepatectomy. I Surg Res 2015; 193:223-228.

54. Liang S, Lai P, Li X, Xu J, Bao Y, Fang Y, et al. Ulinastatin reduces the severity of intestinal damage in the neonatal rat model of necrotizing enterocolitis. Med Sci Monit 2019;25: 9123-9130.

55. Wang WK, Lu QH, Wang X, Wang B, Wang J, Gong HP, et al. Ulinastatin attenuates diabetes-induced cardiac dysfunction by the inhibition of inflammation and apoptosis. Exp Ther Med 2017; 14:2497-2504.

56. Honda Y, Yanagawa Y, Terazumi K, Nishi K. A case of successful treatment of a patient with hyperglycemia of 2700 mg/dL. Am J Emerg Med 2012;30:254.e1-2.

57. Yano T, Anraku S, Nakayama R, Ushijima K. Neuroprotective effect of urinary trypsin inhibitor against focal cerebral ischemia-reperfusion injury in rats. Anesthesiology 2003;98:465-473.

58. Zhao J, Bi W, Xiao S, Lan X, Cheng X, Zhang J, et al. Neuroinflammation induced by lipopolysaccharide causes cognitive impairment in mice. Sci Rep 2019;9:5790.

59. Shikimi T, Hattori K, Takaori S. Existence of a human urinary trypsin inhibitor (urinastatin)-like substance in the rat brain. Jpn J Pharmacol 1992;60:97-103.

60. Li Y, Zhao L, Fu H, Wu Y, Wang T. Ulinastatin suppresses lipopolysaccharide induced neuro-inflammation through the downregulation of nuclear factor- $\kappa B$ in SD rat hippocampal astrocyte. Biochem Biophys Res Commun 2015;458:763-770.

61. Guo W, Li Z, Xie X, Qin T, Wu Y, Li Z, et al. Urinary Trypsin Inhibitor attenuates acute lung injury by improving endothelial progenitor cells functions. Cell Physiol Biochem 2015;36: 1059-1068.

62. Li G, Li T, Li Y, Cai S, Zhang Z, Zeng Z, et al. Ulinastatin inhibits oxidant-induced endothelial hyperpermeability and apoptotic signaling. Int J Clin Exp Pathol 2014;7:7342-7350.

63. Kudo Y, Egashira T, Yamanaka Y. Protective effect of ulinastatin against liver injury caused by ischemia-reperfusion in rats. Jpn J Pharmacol 1992;60:239-245.

64. Liu B, Huang W, Xiao X, Xu Y, Ma S, Xia Z. Neuroprotective effect of ulinastatin on spinal cord ischemia-reperfusion injury in rabbits. Oxid Med Cell Longev 2015;2015:624819.

65. Song F, Kong F, Zhang H, Zhou Y, Li M. Ulinastatin protects against CVB3-induced acute viral myocarditis through Nrf2 activation. Inflammation 2018;41:803-810.

66. Wang S, Cheng ZY, Chen XJ, Xue HZ. Ulinastatin protects rats with myocardial infarction by activating Nrf2/NOS pathway. 
Eur Rev Med Pharmacol Sci 2018;22:8990-8998.

67. Li X, Su L, Zhang X, Zhang C, Wang L, Li Y, et al. Ulinastatin downregulates TLR4 and NF-kB expression and protects mouse brains against ischemia/reperfusion injury. Neurol Res 2017; 39:367-373.

68. Lee JH, Jeong JK, Park SY. Sulforaphane-induced autophagy flux prevents prion protein-mediated neurotoxicity through AMPK pathway. Neuroscience 2014;278:31-39.

69. Mao L, Yang T, Li X, Lei X, Sun Y, Zhao Y, et al. Protective effects of sulforaphane in experimental vascular cognitive impairment: Contribution of the Nrf2 pathway. I Cereb Blood Flow Metab 2019;39:352-366.

70. Boominathan L. Broccoli for enhanced learning, memory, cognition and longevity: sulforaphane from broccoli functions as a learning/memory/cognitive/longevity-enhancer: sulforaphane, isolated from broccoli among others, increases Tissue inhibitor of metalloproteinases 2 (TIMP2) levels, improves cognition, and decreases age-associated decline in memory and learning, via down regulation of its target genes [Internet]. Pondicherry: Genome-2-Bio-Medicine Discovery Center (GBMD); 2018 Jun 29 [cited at 2018 Jun 29]. Available from: https://genomediscovery.org/2018/06/broccoli-for-enhanced-learningmemory-cognition-and-longevity-sulforaphane-from-broccolifunctions-as-a-learning-memory-cognitive-longevity-enhancersulforaphane-isolated-from-broccoli-among-othe/.

71. Negi G, Kumar A, Sharma SS. Nrf2 and NF-кB modulation by sulforaphane counteracts multiple manifestations of diabetic neuropathy in rats and high glucose-induced changes. Curr Neurovasc Res 2011;8:294-304.

72. Shehatou GS, Suddek GM. Sulforaphane attenuates the deve/opment of atherosclerosis and improves endothelial dysfunction in hypercholesterolemic rabbits. Exp Biol Med (Maywood) 2016;241:426-436.

73. Sotokawauchi A, Ishibashi Y, Matsui T, Yamagishi SI. Aqueous extract of glucoraphanin-rich broccoli sprouts inhibits formation of advanced glycation end products and attenuates inflammatory reactions in endothelial cells. Evid Based Complement Alternat Med 2018;2018:9823141.

74. Uddin MS, Mamun AA, Jakaria M, Thangapandiyan S, Ahmad J, Rahman MA, et al. Emerging promise of sulforaphane-mediated Nrf2 signaling cascade against neurological disorders. Sci Total Environ 2020;707:135624.

75. Itoh K, Chiba T, Takahashi S, Ishii T, Igarashi K, Katoh Y, et al. An Nrf2/small Maf heterodimer mediates the induction of phase // detoxifying enzyme genes through antioxidant response elements. Biochem Biophys Res Commun 1997;236: 313-322.

76. Imhoff BR, Hansen JM. Extracellular redox status regulates Nif2 activation through mitochondrial reactive oxygen species. Biochem J 2009;424:491-500.

77. Birks JS, Harvey RJ. Donepezil for dementia due to Alzheimer's disease. Cochrane Database Syst Rev 2018:6:CD001190. 